\title{
UNSTEADY MHD MIXED CONVECTION FLOW OF A MICROPOLAR FLUID OVER A VERTICAL WEDGE
}

\author{
N.C. ROY \\ Department of Mathematics, University of Dhaka \\ BANGLADESH \\ E-mail: nepal@du.ac.bd \\ R.S.R. GORLA* \\ Department of Mechanical Engineering \\ Purdue University Northwest \\ Westville, IN 46391, U.S.A \\ E-mail:rgorla@pnw.edu
}

\begin{abstract}
An analysis is presented to investigate the unsteady magnetohydrodynamic (MHD) mixed convection boundary-layer flow of a micropolar fluid over a vertical wedge in the presence of thermal radiation and heat generation or absorption. The free-stream velocity and surface temperature are assumed to be oscillating in magnitude but not in the direction of the oncoming flow velocity. The governing equations have been solved by two distinct methods, namely, the finite difference method for the entire frequency range, and the series solution for low frequency range and the asymptotic series expansion method for the high frequency range. Numerical solutions provide a good agreement with the series solutions. The amplitudes of skin friction and couple stress coefficients are found to be strongly dependent on the Richardson number and the vortex viscosity parameter. The Prandtl number, the conduction-radiation parameter, the surface temperature parameter and the pressure gradient parameter significantly affect the amplitudes of skin friction, couple stress and surface heat transfer rates. However, the amplitudes of skin friction coefficient are considerably affected by the magnetic field parameter, whereas the amplitudes of heat transfer rate are appreciably changed with the heat generation or absorption parameter. In addition, results are presented for the transient skin friction, couple stress and heat transfer rate with the variations of the Richardson number, the vortex viscosity parameter, the pressure gradient parameter and the magnetic field parameter
\end{abstract}

Key words: micropolar fluid, unsteady flow, radiation, heat generation.

\section{Introduction}

A mixed convection boundary-layer flow and heat transfer over wedge shaped bodies has been the focus of research due to its occurrence in many industrial and engineering applications such as thermal insulation, crude oil extraction, geothermal systems, ground water pollution, heat exchanger and the storage of nuclear waste, etc [1]. When temperature differences between the wedge surface and the fluid are present, there occur density differences within the fluid. As a result, both the flow and heat transfer are affected by the buoyancy force and this process is known as free convection. When the magnitude of the buoyancy force is high even in the presence of a large surface-fluid temperature gradient and moderate flow velocity, forced and free convection are of comparable order. In this case, the combined effect of forced and free convection becomes important. Sing et al. [1] investigated the behavior of an unsteady mixed convection flow of an incompressible viscous fluid over a vertical wedge with constant suction or injection. Lin and Lin [2] examined the laminar forced convection heat transfer from either an isothermal surface or a uniform flux

\footnotetext{
* To whom correspondence should be addressed
} 
boundary to fluids of any Prandtl number. Watanabe [3] theoretically studied the behavior of incompressible laminar boundary layers in a forced flow over a wedge with uniform suction or injection. He also investigated the mixed convection boundary layer flow with uniform suction or injection on a flat plate [4]. Later, Watanabe et al. [5] extended the work of Watanabe [4] for a mixed convection flow with uniform suction and injection.

Micropolar fluids play an in important role in controlling the conjugate effect of forced and free convection over a wedge because of their non-Newtonian characteristics. This type of fluids contains a large amount of rigid, randomly oriented or spherical particles suspended in a viscous medium which move along with microrotations. Lok et al. [6] mentioned that the fluids consisting of an extremely small amount of polymeric additives significantly reduce the skin friction near a rigid body. Applications may arise in the field of aeronautics and submarine navigation where the main concern is the drag reduction of the airplane and ship. Kim [7] investigated the steady laminar flow of micropolar fluids past a wedge with constant surface temperature while Kim and Kim [8] considered the micropolar fluid boundary layer over a wedge with prescribed constant surface heat flux. They solved the problems numerically and examined the details of the flow and heat transfer characteristics and their dependence on the material properties of the micropolar fluid.

A magnetohydrodynamic (MHD) viscous incompressible fluid can be used either for producing heat by electromagnetic field such as MHD power generators and pumps [9] or to control the heat transfer in the cooling of reactors [10] as well as aerodynamic heating [9]. Ishak et al. [11, 12] investigated the MHD boundary-layer flow of a micropolar fluid past a wedge with constant wall heat flux as well as variable wall temperature. Gorla et al. [13] examined the combined effects of buoyancy and magnetic forces of an electrically conducting micropolar fluid along a hot vertical plate. Bhargava et al. [14] studied the influences of a magnetic field and temperature dependent heat source on a micropolar fluid between two parallel porous plates. Mohammadein and Gorla [9] analyzed the mixed convection flow of a micropolar fluid over a semiinfinite horizontal plate with vectored mass transfer in a transverse magnetic field. Nanousis [15] investigated the influence of an applied magnetic field on the mixed convection boundary-layer flow over a wedge with uniform suction or injection. Kumari et al. [16] carried out numerical solutions to examine the mixed convection flow over a vertical wedge with a magnetic field embedded in a porous medium. The effects of a magnetic field for the free convection flow of a Newtonian fluid past an isothermal wedge were studied by Watanabe and Pop [17].

Vajravelu and Nayfeh [18] analyzed the hydromagnetic convection flow and heat transfer from a cone and a wedge with variable surface temperature and internal heat generation or absorption. Ganapathirao [19] investigated the unsteady mixed convection boundary-layer flow over a vertical wedge with chemical reaction and heat generation or absorption. Yih [20] studied the MHD forced convection flow adjacent to a non-isothermal wedge in the presence of viscous and magnetic dissipations and stress work. Chamkha et al. [21] have generalized the work of Yih [20] by considering the effects of bowling or suction, temperaturedependent heat generation or absorption and thermal radiation. Uddin et al. [22] examined the influence of MHD, thermal radiation and heat generation/absorption on a micropolar fluid flow and heat transfer to a wedge.

The present study has been undertaken in order to investigate the unsteady MHD mixed convection boundary-layer flow of a micropolar fluid over a wedge taking into account the effects of the applied magnetic field, thermal radiation, heat generation/absorption and angle of the wedge surface. The governing equations have been solved using the finite difference method and the series solution for the low frequency range and the asymptotic series expansion method for the high frequency range. The agreement of results between the series solutions and the numerical solutions is good. The results are presented in terms of the amplitudes of skin friction coefficient, couple stress coefficient and heat transfer rate with the variation of the Richardson number, vortex viscosity parameter, Prandtl number, conduction-radiation parameter, surface temperature parameter, pressure gradient parameter, magnetic field parameter and heat generation or absorption parameter. We compared our numerical results for the special case of Newtonian fluids with those of Watanabe et al. [5] and Kumari et al. [16] and found them to be in excellent agreement. 


\section{Mathematical formalisms}

We consider a two-dimensional, unsteady, magneto-hydrodynamic, mixed convection boundary layer flow of a micropolar fluid over the surface of a vertical wedge having an angle $\pi \varphi$ in the presence of thermal radiation and heat generation or absorption. The flow configuration and coordinate system are shown in Fig.1. Under the usual boundary layer approximation, the equations for the conservation of mass, momentum, angular velocity and energy may be written as

$$
\begin{aligned}
& \frac{\partial u}{\partial x}+\frac{\partial v}{\partial y}=0 \\
& \frac{\partial u}{\partial t}+u \frac{\partial u}{\partial x}+v \frac{\partial u}{\partial y}=\frac{\partial U}{\partial t}+U \frac{\partial U}{\partial x}+\frac{\mu+\kappa}{\rho} \frac{\partial^{2} u}{\partial y^{2}}+\frac{\kappa}{\rho} \frac{\partial N}{\partial y}+g \beta\left(T-T_{\infty}\right) \cos \left(\frac{\pi \varphi}{2}\right)-\frac{\sigma_{c} B_{0}^{2}}{\rho} u \\
& \frac{\partial N}{\partial t}+u \frac{\partial N}{\partial x}+v \frac{\partial N}{\partial y}=\frac{\gamma}{\rho j} \frac{\partial^{2} N}{\partial y^{2}}-\frac{\kappa}{\rho j}\left(2 N+\frac{\partial u}{\partial y}\right) \\
& \frac{\partial T}{\partial t}+u \frac{\partial T}{\partial x}+v \frac{\partial T}{\partial y}=\alpha \frac{\partial^{2} T}{\partial y^{2}}-\frac{\alpha}{\kappa_{\chi}} \frac{\partial q_{r}}{\partial y}+\frac{Q_{0}}{\rho c_{p}}\left(T-T_{\infty}\right)
\end{aligned}
$$

Here $x$ and $y$ are the coordinates parallel and perpendicular to the wedge surface, $u$ and $v$ are the velocity components in the $x$ - and $y$-directions, respectively, $\mu$ is the viscosity of the fluid, $\rho$ is the density of the fluid, $k$ is the coefficient of gyro-viscosity (or vortex viscosity), $g$ is the acceleration due to gravity, $\beta$ is the coefficient of volumetric expansion, $j$ is the micro-inertia density, $N$ is the component of the microrotation vector normal to the $x y$-plane, $\gamma$ is the spin gradient viscosity, $\alpha$ is the thermal diffusivity, $\kappa_{c}$ is the thermal conductivity, $c_{p}$ is the heat capacity of the fluid, $T$ is the temperature of the fluid in the boundary layer, $\sigma_{c}$ is the electrical conductivity, $Q_{0}$ is the heat generation $(>0)$ or absorption $(<0)$ coefficient while $Q_{0}\left(T-T_{\infty}\right)$ represents the volumetric rate of heat generation/absorption from a source or sink of heat within the fluid. Furthermore, $B_{0}$ is the strength of the magnetic field which is presumed to be applied in the positive $y$ direction and normal to the surface. In this study, we have considered that $B_{0}$ is proportional to $x^{-1 / 4}$.

In Eq.(2.4), the term $q_{r}$ represents the radiative heat-flux in the $y$-direction. The fluid is assumed to be an optically thick gray viscous fluid which can only absorb and emit radiation. In this regard, the radiative heat-flux term is simplified using the Rosseland diffusion approximation and expressed following Raptis $[23]$ as

$$
q_{r}=-\frac{4 \sigma^{*}}{3 k^{*}} \frac{\partial T^{4}}{\partial y}
$$

where $\sigma^{*}$ is the Stefan-Boltzman constant and $k^{*}$ is the Rosseland mean absorption coefficient. Moreover, the spin-gradient viscosity, $\gamma$, introduced in Eq.(2.3) gives a relationship between the coefficients of viscosity and micro-inertia as below

$$
\gamma=\left(\mu+\frac{\kappa}{2}\right) j
$$

The associated boundary conditions are 


$$
\begin{aligned}
& u=v=0, N=-\frac{1}{2} \frac{\partial u}{\partial y}, T=T_{\infty}+\left(T_{w}-T_{\infty}\right)\left(1+\varepsilon e^{i \omega t}\right) \quad \text { at } y=0 \\
& u=U(x, t), \quad v=0, \quad N=0, \quad T=T_{\infty} \text { as } y \rightarrow \infty
\end{aligned}
$$

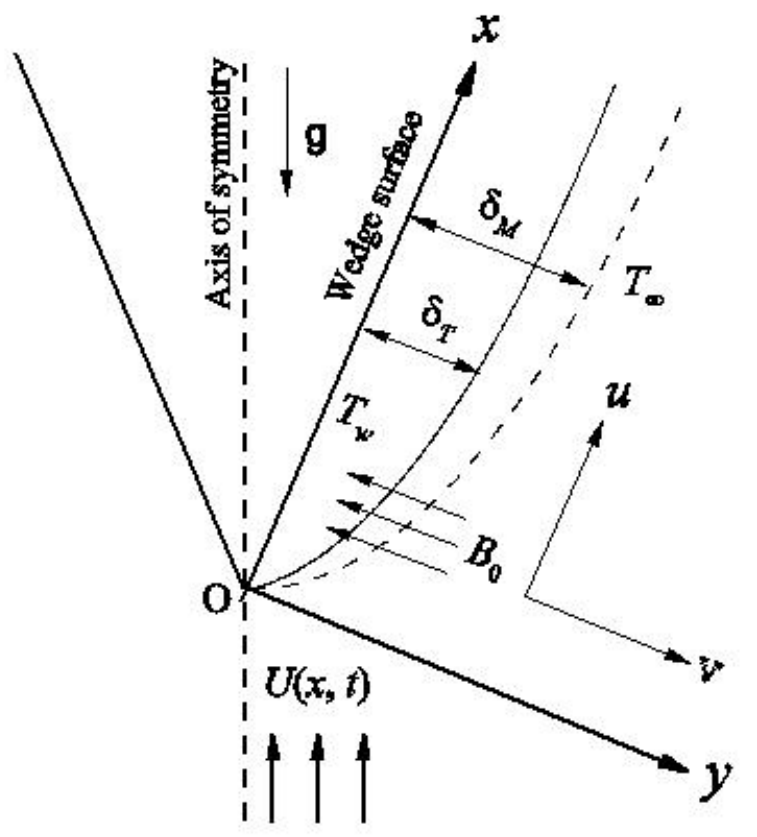

Fig.1. Flow configuration and coordinate system.

In Eqs (2.8), $U(x, t)$ is the local velocity of the mainstream at the outer edge of the boundary layer and is assumed to be of the form

$$
U(x, t)=U_{0} x^{m}\left(1+\varepsilon e^{i \omega t}\right)
$$

where $U_{0}, \varepsilon<<1$ are constants, $\omega$ is the frequency of oscillation and $m$ is the pressure gradient parameter which is related to the angle of wedge $\pi \varphi$ by $m=\varphi /(2-\varphi)$.

We now define the stream function $\psi$ by

$$
u=\frac{\partial \psi}{\partial y} \text { and } v=-\frac{\partial \psi}{\partial x}
$$

which satisfy the continuity Eq.(2.1).

The boundary conditions (2.7) and (2.8) suggest that the functions $\psi, N$ and $T$ may be expressed in the following forms

$$
\begin{aligned}
& \psi(x, y, t)=\psi_{s}(x, y)+\varepsilon \psi_{1}(x, y) e^{i \omega t}, \\
& N(x, y, t)=N_{s}(x, y)+\varepsilon N_{l}(x, y) e^{i \omega t},
\end{aligned}
$$




$$
T(x, y, t)=T_{s}(x, y)+\varepsilon T_{1}(x, y) e^{i \omega t},
$$

where $\psi_{s}, N_{s}, T_{s}$ are the steady-state parts and $\psi_{l}, N_{l}, T_{l}$ are the oscillating parts of the corresponding functions.

Now, using Eqs (2.9)-(2.11) in Eqs (2.2)-(2.4) and equating the coefficients of $\varepsilon^{0}$ give

$$
\begin{aligned}
& \frac{\partial \psi_{s}}{\partial y} \frac{\partial^{2} \psi_{s}}{\partial x \partial y}-\frac{\partial \psi_{s}}{\partial x} \frac{\partial^{2} \psi_{s}}{\partial y^{2}}=m U_{0}{ }^{2} x^{2 m-1}+\frac{\mu+\kappa}{\rho} \frac{\partial^{3} \psi_{s}}{\partial y^{3}}+\frac{\kappa}{\rho} \frac{\partial N_{s}}{\partial y}+ \\
& +g \beta\left(T_{s}-T_{\infty}\right) \cos \left(\frac{\pi \varphi}{2}\right)-\frac{\sigma_{c} B_{0}^{2}}{\rho} \frac{\partial \psi_{s}}{\partial y} \\
& \frac{\partial \psi_{s}}{\partial y} \frac{\partial N_{s}}{\partial x}-\frac{\partial \psi_{s}}{\partial x} \frac{\partial N_{s}}{\partial y}=\frac{\gamma}{\rho j} \frac{\partial^{2} N_{s}}{\partial y^{2}}-\frac{\kappa}{\rho j}\left(2 N_{s}+\frac{\partial^{2} \psi_{s}}{\partial y^{2}}\right), \\
& \frac{\partial \psi_{s}}{\partial y} \frac{\partial T_{s}}{\partial x}-\frac{\partial \psi_{s}}{\partial x} \frac{\partial T_{s}}{\partial y}=\alpha \frac{\partial^{2} T_{s}}{\partial y^{2}}+\frac{16 \alpha \sigma}{3 \kappa_{c}\left(a+\sigma_{s}\right)} \frac{\partial}{\partial y}\left(T_{s}^{3} \frac{\partial T_{s}}{\partial y}\right)+\frac{Q_{0}}{\rho c_{p}}\left(T_{s}-T_{\infty}\right)
\end{aligned}
$$

The set of equations for $\psi_{s}, N_{s}$ and $T_{s}$ represents the steady-state solutions that can be determined by the following functions

$$
\begin{aligned}
& \psi_{s}=\left(\frac{2}{m+1} v U_{0}\right)^{1 / 2} x^{\frac{m+1}{2}} f(\eta), \quad N_{s}=U_{0}\left(\frac{U_{0}}{v} \frac{m+1}{2}\right)^{1 / 2} x^{\frac{3 m-1}{2}} h(\eta), \\
& T_{s}=T_{\infty}+\left(T_{w}-T_{\infty}\right) \theta(\eta), \quad \eta=\left(\frac{U_{0}}{v} \frac{m+1}{2}\right)^{1 / 2} x^{\frac{m-1}{2}} y, \quad j=\left(\frac{v}{U_{0}}\right) x^{1-m} .
\end{aligned}
$$

Substituting Eqs (2.15) into Eqs (2.12)-(2.14), we obtain the following dimensionless equations

$$
\begin{aligned}
& (1+K) f^{\prime \prime \prime}+f f^{\prime \prime}+\frac{2 m}{m+1}\left(1-f^{\prime 2}\right)+K h^{\prime}+\frac{2}{m+1}\left(R i \theta-M f^{\prime}\right)=0, \\
& \left(1+\frac{K}{2}\right) h^{\prime \prime}+f h^{\prime}-\frac{3 m-1}{m+1} f^{\prime} h-\frac{2 K}{m+1}\left(2 h+f^{\prime \prime}\right)=0, \\
& \frac{1}{\operatorname{Pr}}\left\{1+\frac{4}{3} R_{d}(1+\Delta \theta)^{3}\right\} \theta^{\prime \prime}+\frac{4 \Delta}{\operatorname{Pr}} R_{d}(1+\Delta \theta)^{2} \theta^{\prime 2}+f \theta^{\prime}+\frac{2 \Omega}{m+1} \theta=0,
\end{aligned}
$$

subject to the boundary conditions

$$
\begin{aligned}
& f=f^{\prime}=0, \quad h=-\frac{1}{2} f^{\prime \prime}, \quad \theta=1 \quad \text { at } \quad \eta=0, \\
& f^{\prime}=1, \quad h=0, \quad \theta=0 \quad \text { as } \quad \eta \rightarrow \infty .
\end{aligned}
$$


In the above equations $\mathrm{Ri}=\mathrm{Gr}_{x} / \mathrm{Re}_{x}^{2}$ is the Richardson number, $\mathrm{Gr}_{x}=g \beta\left(T_{w}-T_{\infty}\right) x^{3} \cos (\pi \varphi / 2) / v^{2}$ is the Grashof number, $\operatorname{Re}_{x}=U_{0} x^{m+1} / v$ is the Reynolds number, $M=\sigma_{c} B_{0}^{2} x^{1-m} / \rho U_{0}$ is the magnetic field parameter, $\operatorname{Pr}=v / \alpha$ is the Prandtl number, $K=\kappa / \mu$ is the vortex viscosity parameter, $R_{d}=4 \sigma T_{\infty}^{3} / \kappa_{c}\left(a+\sigma_{s}\right)$ is the conduction-radiation parameter, $\Delta=T_{w} / T_{\infty}-1$ is the surface temperature parameter and $\Omega=v Q_{0} x^{1-m} / \rho c_{p} U_{0}$ is the heat generation $(>0)$ or absorption $(<0)$ parameter.

Using expressions (2.9)-(2.11) in Eqs (2.2)-(2.4) and equating the coefficients of $\varepsilon$, we obtain equations for time-dependent components $\psi_{l}, N_{l}$ and $T_{l}$ as

$$
\begin{aligned}
& i \omega \frac{\partial \psi_{1}}{\partial y}+\frac{\partial \psi_{0}}{\partial y} \frac{\partial^{2} \psi_{1}}{\partial x \partial y}+\frac{\partial \psi_{1}}{\partial y} \frac{\partial^{2} \psi_{0}}{\partial x \partial y}-\frac{\partial \psi_{0}}{\partial x} \frac{\partial^{2} \psi_{1}}{\partial y^{2}}-\frac{\partial \psi_{1}}{\partial x} \frac{\partial^{2} \psi_{0}}{\partial y^{2}}= \\
& =i \omega U_{0} x^{m}+2 m U_{0}{ }^{2} x^{2 m-1}+\frac{\mu+\kappa}{\rho} \frac{\partial^{3} \psi_{1}}{\partial y^{3}}+\frac{\kappa}{\rho} \frac{\partial N_{1}}{\partial y}+g \beta T_{1} \cos \left(\frac{\pi \varphi}{2}\right)-\frac{\sigma_{c} B_{0}^{2}}{\rho} \frac{\partial \psi_{1}}{\partial y} \\
& i \omega N_{1}+\frac{\partial \psi_{0}}{\partial y} \frac{\partial N_{1}}{\partial x}+\frac{\partial \psi_{1}}{\partial y} \frac{\partial N_{0}}{\partial x}-\frac{\partial \psi_{0}}{\partial x} \frac{\partial N_{1}}{\partial y}-\frac{\partial \psi_{1}}{\partial x} \frac{\partial N_{0}}{\partial y}=\frac{\gamma}{\rho j} \frac{\partial^{2} N_{1}}{\partial y^{2}}-\frac{\kappa}{\rho j}\left(2 N_{1}+\frac{\partial^{2} \psi_{1}}{\partial y^{2}}\right) \\
& i \omega T_{1}+\frac{\partial \psi_{0}}{\partial y} \frac{\partial T_{1}}{\partial x}+\frac{\partial \psi_{1}}{\partial y} \frac{\partial T_{0}}{\partial x}-\frac{\partial \psi_{0}}{\partial x} \frac{\partial T_{1}}{\partial y}-\frac{\partial \psi_{1}}{\partial x} \frac{\partial T_{0}}{\partial y}= \\
& =\alpha \frac{\partial^{2} T_{1}}{\partial y^{2}}+\frac{16 \alpha \sigma}{3 \kappa_{c}\left(a+\sigma_{s}\right)} \frac{\partial}{\partial y}\left(T_{0}^{3} \frac{\partial T_{1}}{\partial y}+3 T_{0}^{2} T_{1} \frac{\partial T_{0}}{\partial y}\right)+\frac{Q_{0}}{\rho c_{p}} T_{1}
\end{aligned}
$$

with the boundary conditions

$$
\begin{aligned}
& \psi_{1}=\frac{\partial \psi_{1}}{\partial y}=0, \quad N_{1}=-\frac{1}{2} \frac{\partial^{2} \psi_{1}}{\partial y^{2}}, \quad T_{1}=T_{w}-T_{\infty} \quad \text { at } \quad y=0, \\
& \frac{\partial \psi_{1}}{\partial y}=U_{0} x^{m}, \quad N_{1}=0, \quad T_{1}=0 \quad \text { as } \quad y \rightarrow \infty .
\end{aligned}
$$

To non-dimensionalize Eqs (2.21)-(2.23), we introduce the following expressions

$$
\begin{aligned}
& \Psi_{1}=\left(\frac{2}{m+1} v U_{0}\right)^{1 / 2} x^{\frac{m+1}{2}} F(\xi, \eta), \quad N_{1}=U_{0}\left(\frac{U_{0}}{v} \frac{m+1}{2}\right)^{1 / 2} x^{\frac{3 m-1}{2}} H(\xi, \eta), \\
& T_{1}=\left(T_{w}-T_{\infty}\right) \Theta(\xi, \eta), \quad \xi=\omega x^{1-m} / U_{0} .
\end{aligned}
$$

Substituting Eqs (2.15) and (2.26) into Eqs (2.21)-(2.23), we obtain 


$$
\begin{aligned}
& (1+K) F^{\prime \prime \prime}+\left(f F^{\prime \prime}+f^{\prime \prime} F\right)+\frac{4 m}{m+1}\left(1-f^{\prime} F^{\prime}\right)+\frac{2 i \xi}{m+1}\left(1-F^{\prime}\right)+K H^{\prime}+ \\
& +\frac{2}{m+1}\left(\operatorname{Ri} \Theta-M F^{\prime}\right)=\frac{2(1-m)}{m+1} \xi\left(f^{\prime} \frac{\partial F^{\prime}}{\partial \xi}-f^{\prime \prime} \frac{\partial F}{\partial \xi}\right), \\
& \left(1+\frac{K}{2}\right) H^{\prime \prime}+f H^{\prime}+F h^{\prime}-\frac{3 m-1}{m+1}\left(f^{\prime} H+h F^{\prime}\right)-\frac{2 i \xi}{m+1} H-\frac{2 K}{m+1}\left(2 H+F^{\prime \prime}\right)= \\
& =\frac{2(1-m)}{m+1} \xi\left(f^{\prime} \frac{\partial H}{\partial \xi}-h^{\prime} \frac{\partial F}{\partial \xi}\right), \\
& \frac{1}{\operatorname{Pr}}\left\{1+\frac{4}{3} R_{d}(1+\Delta \theta)^{3}\right\} \Theta^{\prime \prime}+\frac{8 \Delta}{\operatorname{Pr}} R_{d}(1+\Delta \theta)^{2} \theta^{\prime} \Theta^{\prime}+\frac{8 \Delta^{2}}{\operatorname{Pr}} R_{d}(1+\Delta \theta) \theta^{\prime 2} \Theta+ \\
& +\frac{4 \Delta}{\operatorname{Pr}} R_{d}(1+\Delta \theta)^{2} \theta^{\prime \prime} \Theta+F \theta^{\prime}+f \Theta^{\prime}-\frac{2 i \xi}{m+1} \Theta+\frac{2 \Omega}{m+1} \Theta=\frac{2(1-m)}{m+1} \xi\left(f^{\prime} \frac{\partial \Theta}{\partial \xi}-\theta^{\prime} \frac{\partial F}{\partial \xi}\right) .
\end{aligned}
$$

The associated boundary conditions for Eqs (2.27)-(2.29) are

$$
\begin{aligned}
& F=F^{\prime}=0, \quad H=-\frac{1}{2} F^{\prime \prime}, \quad \Theta=1 \quad \text { at } \quad \eta=0, \\
& F^{\prime}=1, \quad H=0, \quad \Theta=0 \quad \text { as } \quad \eta \rightarrow \infty .
\end{aligned}
$$

The values of the physical quantities, namely, the local skin friction, couple stress and heat transfer rate at the surface of the wedge, are readily obtained from the solutions of the sets of Eqs (2.16)-(2.20) and (2.27)-(2.31). These are important not only from the physical point of view but also from the experimental point of view. The local skin friction coefficient, $C_{f}$, couple stress coefficient, $m_{N}$ and heat transfer rate (Nusselt number) $\mathrm{Nu}$ may be obtained by the relations given below

$$
\begin{aligned}
& C_{f}=\frac{2(\mu+\kappa)}{\rho U_{\infty}^{2}}\left(\frac{\partial u_{1}}{\partial y}\right)_{y=0}=(1+K)\{2(m+1)\}^{1 / 2} \operatorname{Re}_{x}^{-1 / 2} F^{\prime \prime}(\xi, 0), \\
& m_{N}=\frac{x \gamma}{j \rho U_{\infty}^{2}}\left(\frac{\partial N}{\partial y}\right)_{y=0}=\left(1+\frac{K}{2}\right) \frac{m+1}{2} H^{\prime}(\xi, 0), \\
& \mathrm{Nu}=-\frac{x k_{c}}{k_{c}\left(T_{w}-T_{\infty}\right)}\left(\frac{\partial T}{\partial y}\right)_{y=0}=-\left(\frac{m+1}{2}\right)^{1 / 2} \operatorname{Re}_{x}^{1 / 2} \Theta^{\prime}(\xi, 0) .
\end{aligned}
$$

In this study, the results will be presented in terms of the amplitudes of the skin friction coefficient, $A_{u}$, couple stress coefficient, $A_{m}$ and heat transfer coefficient, $A_{q}$ given by the expressions

$$
A_{u}=\left|F^{\prime \prime}(\xi, 0)\right|=\sqrt{\left\{F_{r}^{\prime \prime}(\xi, 0)\right\}^{2}+\left\{F_{i}^{\prime \prime}(\xi, 0)\right\}^{2}},
$$




$$
\begin{aligned}
& A_{m}=\left|H^{\prime}(\xi, 0)\right|=\sqrt{\left\{H_{r}^{\prime}(\xi, 0)\right\}^{2}+\left\{H_{i}^{\prime}(\xi, 0)\right\}^{2}}, \\
& A_{q}=\left|\Theta^{\prime}(\xi, 0)\right|=\sqrt{\left\{\Theta_{r}^{\prime}(\xi, 0)\right\}^{2}+\left\{\Theta_{i}^{\prime}(\xi, 0)\right\}^{2}}
\end{aligned}
$$

where $\left(F_{r}^{\prime \prime}, F_{i}^{\prime \prime}\right),\left(H_{r}^{\prime}, H_{i}^{\prime}\right)$ and $\left(\Theta_{r}^{\prime}, \Theta_{i}^{\prime}\right)$ are the corresponding real and imaginary parts of the gradients of the axial velocity, angular velocity and temperature at the surface.

\section{Solution methodologies}

To find the solutions of Eqs (2.16)-(2.20) representing the steady mean flow and temperature fields, we have employed the finite difference method for different values of the physical parameters $m, \operatorname{Pr}, \mathrm{Ri}, K$, $R_{d}, M, \Omega$ and $\Delta$. Subsequently, the resulting solutions are applied in finding the solutions of Eqs (2.27)-(2.31) that provide the oscillating parts of the flow and the temperature fields. In order to verify the numerical solutions, Eqs (2.27)-(2.29) satisfying the boundary conditions (2.30)-(2.31) are also solved using the series solutions for the low frequency range $(\xi<<1)$ and the asymptotic series expansion method for the high frequency range $(\xi>>1)$. Details of the solutions are discussed in the following sections.

\subsection{Series solutions for small $\xi(<<1)$}

As the flow and heat transfer near the leading edge are usually determined for a very small range of frequencies, the results will be based on the finite number of terms in a series. Thus, we seek the solutions of the unknown functions $F, H$ and $\Theta$ of Eqs (2.27)-(2.29) by the following series expansions

$$
F(\xi, \eta)=\sum_{n=0}^{\infty}(2 i \xi)^{n} F_{n}(\eta), H(\xi, \eta)=\sum_{n=0}^{\infty}(2 i \xi)^{n} H_{n}(\eta), \Theta(\xi, \eta)=\sum_{n=0}^{\infty}(2 i \xi)^{n} \Theta_{n}(\eta)
$$

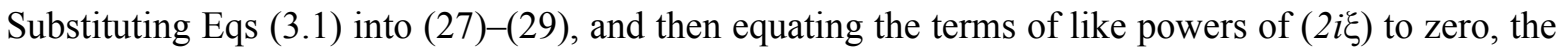
following groups of ordinary differential equations are obtained:

For $n=0$,

$$
\begin{aligned}
& (1+K) F_{0}^{\prime \prime \prime}+f F_{0}^{\prime \prime}+f^{\prime \prime} F_{0}+\frac{4 m}{m+1}\left(1-f^{\prime} F_{0}^{\prime}\right)+K H_{0}^{\prime}+\frac{2}{m+1}\left(\mathrm{Ri}_{0}-M F_{0}^{\prime}\right)=0, \\
& \left(1+\frac{K}{2}\right) H_{0}^{\prime \prime}+f H_{0}^{\prime}+F_{0} h^{\prime}-\frac{3 m-1}{m+1}\left(f^{\prime} H_{0}+h F_{0}^{\prime}\right)-\frac{2 K}{m+1}\left(2 H_{0}+F_{0}^{\prime \prime}\right)=0, \\
& \frac{1}{\operatorname{Pr}}\left\{1+\frac{4}{3} R_{d}(1+\Delta \theta)^{3}\right\} \Theta_{0}^{\prime \prime}+\frac{8 \Delta}{\operatorname{Pr}} R_{d}(1+\Delta \theta)^{2} \theta^{\prime} \Theta_{0}^{\prime}+\frac{8 \Delta^{2}}{\operatorname{Pr}} R_{d}(1+\Delta \theta) \theta^{\prime 2} \Theta_{0}+ \\
& +\frac{4 \Delta}{\operatorname{Pr}} R_{d}(1+\Delta \theta)^{2} \theta^{\prime \prime} \Theta_{0}+F_{0} \theta^{\prime}+f \Theta_{0}^{\prime}+\frac{2 \Omega}{m+1} \Theta_{0}=0,
\end{aligned}
$$

For $n=1$,

$$
(1+K) F_{1}^{\prime \prime \prime}+f F_{1}^{\prime \prime}+\frac{3-m}{m+1} f^{\prime \prime} F_{1}-2 f^{\prime} F_{1}^{\prime}+K H_{1}^{\prime}+\frac{2}{m+1}\left(\mathrm{Ri}_{1}-M F_{1}^{\prime}\right)+\frac{1}{m+1}=\frac{1}{m+1} F_{0}^{\prime},
$$




$$
\begin{aligned}
& \left(1+\frac{K}{2}\right) H_{l}^{\prime \prime}+f H_{1}^{\prime}+\frac{3-m}{m+1} F_{1} h^{\prime}-\frac{3 m-1}{m+1} h F_{1}^{\prime}-\frac{m+1}{2} f^{\prime} H_{1}-\frac{2 K}{m+1}\left(2 H_{1}+F_{l}^{\prime \prime}\right)=\frac{1}{m+1} H_{0} \\
& \frac{1}{\operatorname{Pr}}\left\{1+\frac{4}{3} R_{d}(1+\Delta \theta)^{3}\right\} \Theta_{1}^{\prime \prime}+\frac{8 \Delta}{\operatorname{Pr}} R_{d}(1+\Delta \theta)^{2} \theta^{\prime} \Theta_{1}^{\prime}+\frac{8 \Delta^{2}}{\operatorname{Pr}} R_{d}(1+\Delta \theta) \theta^{\prime 2} \Theta_{l}+ \\
& +\frac{4 \Delta}{\operatorname{Pr}} R_{d}(1+\Delta \theta)^{2} \theta^{\prime \prime} \Theta_{1}+f \Theta_{l}^{\prime}+\frac{3-m}{m+1} \theta^{\prime} F_{1}-\frac{2(1-m)}{m+1} f^{\prime} \Theta_{1}+\frac{2 \Omega}{m+1} \Theta_{1}=\frac{1}{m+1} \Theta_{0}
\end{aligned}
$$

For $n \geq 2$,

$$
\begin{aligned}
& (1+K) F_{n}^{\prime \prime \prime}+f F_{n}^{\prime \prime}+\left\{1+\frac{2(1-m)}{m+1} n\right\} f^{\prime \prime} F_{n}-\left\{\frac{4 m}{m+1}+\frac{2(1-m)}{m+1} n\right\} f^{\prime} F_{n}^{\prime}+K H_{n}^{\prime}+ \\
& +\frac{2}{m+1}\left(\operatorname{Ri} \Theta_{n}-M F_{n}^{\prime}\right)=\frac{1}{m+1} F_{n-1}^{\prime}, \\
& \left(1+\frac{K}{2}\right) H_{n}^{\prime \prime}+f H_{n}^{\prime}+\left\{1+\frac{2(1-m)}{m+1} n\right\} h^{\prime} F_{n}-\frac{3 m-1}{m+1} h F_{n}^{\prime}+ \\
& -\left\{\frac{3 m-1}{m+1}+\frac{2(1-m)}{m+1} n\right\} f^{\prime} H_{n}-\frac{2}{m+1}\left(2 H_{n}+F_{n}^{\prime \prime}\right)=\frac{1}{m+1} H_{n-1}, \\
& \frac{1}{\operatorname{Pr}}\left\{1+\frac{4}{3} R_{d}(1+\Delta \theta)^{3}\right\} \Theta_{n}^{\prime \prime}+\frac{8 \Delta}{\operatorname{Pr}} R_{d}(1+\Delta \theta)^{2} \theta^{\prime} \Theta_{n}^{\prime}+\frac{8 \Delta^{2}}{\operatorname{Pr}} R_{d}(1+\Delta \theta) \theta^{\prime 2} \Theta_{n}+ \\
& +\frac{4 \Delta}{\operatorname{Pr}} R_{d}(1+\Delta \theta)^{2} \theta^{\prime \prime} \Theta_{n}+f \Theta_{n}^{\prime}+\left\{1+\frac{2(1-m)}{m+1} n\right\} \theta^{\prime} F_{n}-\frac{2(1-m)}{m+1} n f^{\prime} \Theta_{n}+ \\
& +\frac{2 \Omega}{m+1} \Theta_{n}=\frac{1}{m+1} \Theta_{n-1} .
\end{aligned}
$$

The corresponding boundary conditions are

$$
\begin{aligned}
& F_{n}(0)=F_{n}^{\prime}(0)=\Theta_{n}(0)=0, \quad H_{n}^{\prime}(0)=-\frac{1}{2} F_{n}^{\prime \prime}(0), \quad \Theta_{0}(0)=1 \quad \text { at } \quad \eta=0, \\
& F_{n}^{\prime}(\infty)=1, \quad H_{n}^{\prime}(\infty)=0, \quad \Theta_{n}(\infty)=0 \quad \text { as } \quad \eta \rightarrow \infty .
\end{aligned}
$$

Here primes denote derivatives with respect to $\eta$ only.

Equations (3.2)-(3.10) are linear but coupled. Hence these are solved independently group-wise successively. The implicit Runge-Kutta-Butcher [24] initial value solver together with Nachtsheim-Swigert [25] iteration scheme is employed to solve Eqs (3.2)-(3.10) up to $O\left(\xi^{10}\right)$.

\subsection{Asymptotic solutions for large $\xi(>>1)$}

In this section, attention will be given to the behavior of the solutions of Eqs (2.27)-(2.29) when $\xi$ is large. When the frequency of oscillation $\omega$ is too large, the boundary layer response might be significant in a 
very thin region adjacent to the surface. Consequently, the solutions become independent of $\xi$ provided that the frequency of oscillation $\omega$ tends to infinity. Therefore we introduce the following transformations in Eqs (2.27)-(2.29)

$$
F(\xi, \eta)=\xi^{-1 / 2} \phi(\xi, Y), H(\xi, \eta)=\xi^{1 / 2} g(\xi, Y), \Theta(\xi, \eta)=\Theta(\xi, Y), \quad Y=\xi^{1 / 2} \eta
$$

Thus Eqs (2.27)-(2.29) become

$$
\begin{aligned}
& (1+K) \frac{\partial^{3} \phi}{\partial Y^{3}}+\xi^{-1 / 2} f \frac{\partial^{2} \phi}{\partial Y^{2}}+\frac{2}{m+1} \xi^{-3 / 2} f^{\prime \prime} \phi+\frac{4 m}{m+1} \xi^{-1}\left\{1-f^{\prime} \frac{\partial \phi}{\partial Y}\right\}+ \\
& +\frac{2 i}{m+1}\left\{1-\frac{\partial \phi}{\partial Y}\right\}+K \frac{\partial g}{\partial Y}+\frac{2}{m+1} \xi^{-1}\left(\operatorname{Ri} \Theta-M \frac{\partial \phi}{\partial Y}\right)= \\
& =\frac{2(1-m)}{m+1}\left\{f^{\prime}\left(\frac{\partial^{2} \phi}{\partial Y \partial \xi}+\frac{Y}{2 \xi} \frac{\partial^{2} \phi}{\partial Y^{2}}\right)-\xi^{-1 / 2} f^{\prime \prime}\left(\frac{\partial \phi}{\partial \xi}+\frac{Y}{2 \xi} \frac{\partial \phi}{\partial Y}\right)\right\} \\
& \left(1+\frac{K}{2}\right) \frac{\partial^{2} g}{\partial Y^{2}}+\xi^{-1 / 2} f \frac{\partial g}{\partial Y}+\frac{2}{m+1} \xi^{-2} h^{\prime} \phi-\frac{3 m-1}{m+1} \xi^{-1 / 2} h \frac{\partial g}{\partial Y}-\frac{2 m}{m+1} \xi^{-1} f^{\prime} g-\frac{2 i}{m+1} g+ \\
& -\frac{2 K}{m+1} \xi^{-1}\left(2 g+\frac{\partial^{2} \phi}{\partial Y^{2}}\right)=\frac{2(1-m)}{m+1}\left\{f^{\prime}\left(\frac{\partial g}{\partial \xi}+\frac{Y}{2 \xi} \frac{\partial g}{\partial Y}\right)-\xi^{-1} h^{\prime}\left(\frac{\partial \phi}{\partial \xi}+\frac{Y}{2 \xi} \frac{\partial \phi}{\partial Y}\right)\right\} \\
& \frac{1}{\operatorname{Pr}}\left\{1+\frac{4}{3} R_{d}(1+\Delta \theta)^{3}\right\} \frac{\partial^{2} \Theta}{\partial Y^{2}}+\frac{8 \Delta}{\operatorname{Pr}} R_{d}(1+\Delta \theta)^{2} \theta^{\prime} \xi^{-1 / 2} \frac{\partial \Theta}{\partial Y}+\frac{8 \Delta^{2}}{\operatorname{Pr}} R_{d}(1+\Delta \theta)\left(\theta^{\prime}\right)^{2} \xi^{-1} \Theta+ \\
& +\frac{4 \Delta}{\operatorname{Pr}} R_{d}(1+\Delta \theta)^{2} \theta^{\prime \prime} \xi^{-1} \Theta+\xi^{-1 / 2} f \frac{\partial \Theta}{\partial Y}+\frac{2}{m+1} \xi^{-3 / 2} \theta^{\prime} \phi-\frac{2 i}{m+1} \Theta+\frac{2 \Omega}{m+1} \xi^{-1} \Theta= \\
& =\frac{2(1-m)}{m+1}\left\{f^{\prime}\left(\frac{\partial \Theta}{\partial \xi}+\frac{Y}{2 \xi} \frac{\partial \Theta}{\partial Y}\right)-\xi^{-1 / 2} \theta^{\prime}\left(\frac{\partial \phi}{\partial \xi}+\frac{Y}{2 \xi} \frac{\partial \phi}{\partial Y}\right)\right\} .
\end{aligned}
$$

For small values of $\eta$, we can expand the solutions of Eqs (2.16)-(2.18) in the power series

$$
\begin{aligned}
& f=a_{2} \eta^{2}+a_{3} \eta^{3}+a_{4} \eta^{4}+a_{5} \eta^{5}+\cdots, \\
& h=b_{0}+b_{1} \eta+b_{2} \eta^{2}+b_{3} \eta^{3}+b_{4} \eta^{4}+b_{5} \eta^{5}+\cdots \\
& \theta=1+c_{1} \eta+c_{2} \eta^{2}+c_{3} \eta^{3}+c_{4} \eta^{4}+c_{5} \eta^{5}+\cdots
\end{aligned}
$$

Using Eqs (3.17)-(3.19) into Eqs (2.16)-(2.20), we get

$$
a_{2}=\frac{1}{2} f^{\prime \prime}(0), b_{0}=h(0)=-\frac{1}{2} f^{\prime \prime}(0), b_{1}=h^{\prime}(0), c_{1}=\theta^{\prime}(0), \cdots \cdots
$$

Now the solutions of Eqs (3.14)-(3.16) can be obtained in the following forms 


$$
\phi(\xi, Y)=\sum_{n=0}^{\infty} \xi^{-n / 2} E_{n}(Y), g(\xi, Y)=\sum_{n=0}^{\infty} \xi^{-n / 2} J_{n}(Y) \text { and } \Theta(\xi, Y)=\sum_{n=0}^{\infty} \xi^{-n / 2} L_{n}(Y)
$$

Substituting (3.21) into Eqs (3.14)-(3.16), and equating the like powers of $\xi$, we obtain

$$
\begin{aligned}
& (1+K) E_{0}^{\prime \prime \prime}-\frac{2}{m+1} i E_{0}^{\prime}=-\left(\frac{2 i}{m+1}+K J_{0}^{\prime}\right), \\
& (1+K) E_{1}^{\prime \prime \prime}-\frac{2}{m+1} i E_{1}^{\prime}=-K J_{1}^{\prime}, \\
& (1+K) E_{2}^{\prime \prime \prime}-\frac{2}{m+1} i E_{2}^{\prime}=-\left(\frac{4 m}{m+1}+\frac{2 \mathrm{Ri}}{m+1} L_{0}-\frac{2 M}{m+1} E_{0}^{\prime}+K J_{2}^{\prime}\right), \\
& \left(1+\frac{K}{2}\right) J_{0}^{\prime \prime}-\frac{2 i}{m+1} J_{0}=0, \\
& \left(1+\frac{K}{2}\right) J_{1}^{\prime \prime}-\frac{2 i}{m+1} J_{1}=\frac{b_{0}(3 m-1)}{m+1}, \\
& \left(1+\frac{K}{2}\right) J_{2}^{\prime \prime}-\frac{2 i}{m+1} J_{2}=\frac{4}{m+1} K J_{0}+\frac{b_{1}(3 m-1)}{m+1} Y J_{0}^{\prime}+\frac{b_{0}(3 m-1)}{m+1} J_{1}^{\prime}+\frac{2 K}{m+1} E_{0}^{\prime \prime},
\end{aligned}
$$

and

$$
\begin{aligned}
& \left\{1+\frac{4}{3} R_{d}(1+\Delta)^{3}\right\} L_{0}^{\prime \prime}-\frac{2 i \operatorname{Pr}}{m+1} L_{0}=0, \\
& \left\{1+\frac{4}{3} R_{d}(1+\Delta)^{3}\right\} L_{1}^{\prime \prime}-\frac{2 i \operatorname{Pr}}{m+1} L_{1}=-G_{1}\left(Y L_{0}^{\prime \prime}+2 L_{0}^{\prime}\right), \\
& \left\{1+\frac{4}{3} R_{d}(1+\Delta)^{3}\right\} L_{2}^{\prime \prime}-\frac{2 i \operatorname{Pr}}{m+1} L_{2}=-\left\{G_{1}\left(Y L_{1}^{\prime \prime}+2 L_{1}^{\prime}\right)+G_{2}\left(Y^{2} L_{0}^{\prime \prime}+4 Y L_{0}^{\prime}+2 L_{0}\right)+\frac{2 \Omega \operatorname{Pr}}{m+1} L_{0}\right\} .
\end{aligned}
$$

The corresponding boundary conditions are

$$
\begin{aligned}
& E_{n}(0)=E_{n}^{\prime}(0)=0, \quad E_{0}^{\prime}(\infty)=(1+\operatorname{Pr})^{1 / 2}, \quad E_{n}^{\prime}(\infty)=0, \text { for } n=0,1, \ldots, \\
& J_{0}(0)=-\frac{1}{2} E_{n}^{\prime \prime}(0), J_{n}(0)=J_{n}(\infty)=0, \text { for } n=1,2, \ldots, \\
& L_{0}(0)=1, \quad L_{n}(0)=L_{n}(\infty)=0, \text { for } n=1,2, \ldots
\end{aligned}
$$


Solving the above equations, we can find the expressions for $F^{\prime \prime}(\xi, 0), H^{\prime}(\xi, 0)$ and $\Theta^{\prime}(\xi, 0)$ as

$$
\begin{aligned}
& F^{\prime \prime}(\xi, 0)=\sum_{n=0}^{\infty} \xi^{(1-n) / 2} E_{n}^{\prime \prime}(0), \\
& H^{\prime}(\xi, 0)=\sum_{n=0}^{\infty} \xi^{(2-n) / 2} J_{n}^{\prime}(0), \\
& \Theta^{\prime}(\xi, 0)=\sum_{n=0}^{\infty} \xi^{(1-n) / 2} L_{n}{ }^{\prime}(0)
\end{aligned}
$$

where

with

$$
\begin{aligned}
& E_{0}{ }^{\prime \prime}(0)=\frac{2 \sqrt{m_{1}} D_{3} r}{D_{2}}, \quad E_{1}^{\prime \prime}(0)=-\frac{b_{0} m_{4} K}{D_{2}^{2}}, \quad E_{2}^{\prime \prime}(0)=\frac{D_{5}}{D_{6}}, \cdots, \\
& J_{0}^{\prime \prime}(0)=\frac{m_{1} D_{3} r^{2}}{\sqrt{K_{2}} D_{2}}, \quad J_{1}^{\prime \prime}(0)=-\frac{b_{0} \sqrt{m_{1}} m_{4} \sqrt{K_{1}}\left(2 D_{3}^{2}-K\right) r}{2 D_{1}^{2}}, \quad J_{2}^{\prime \prime}(0)=\frac{D_{13}}{D_{14}}, \cdots, \\
& L_{0}{ }^{\prime}(0)=-\frac{s \sqrt{m_{1}}}{\sqrt{G_{0}}}, \quad L_{1}^{\prime}(0)=-\frac{3 G_{1}}{4 G_{0}}, \quad L_{2}{ }^{\prime}(0)=\frac{-3 G_{1}^{2}+8 G_{0}\left(-3 G_{2}+2 G_{3}\right)}{32 \sqrt{m_{1}} G_{0}^{3 / 2} s}, \cdots
\end{aligned}
$$

$$
\begin{aligned}
& r=\sqrt{i}, \quad s=\sqrt{i \operatorname{Pr}}, \quad m_{1}=2 /(m+1), \quad m_{2}=2 m /(m+1), \\
& m_{3}=(1-m) /(m+1), \quad m_{4}=(3 m-1) /(m+1), \quad K_{1}=1+K, \quad K_{2}=1+K / 2, \\
& G_{0}=1+\frac{4}{3} R_{d}(1+\Delta \theta)^{3}, \quad G_{1}=4 \Delta R_{d}(1+\Delta)^{2} c_{1}, \quad G_{2}=4 R_{d} \Delta(1+\Delta)\left\{c_{1}^{2}+c_{2}(1+\Delta)\right\}, \\
& G_{3}=2 G_{1}+m_{1} \Omega \operatorname{Pr}, \quad D_{1}=2\left(K_{1}-\sqrt{K_{1} K_{2}}\right)-K, \\
& D_{2}=2\left(K_{1}+\sqrt{K_{1} K_{2}}\right)-K, \quad D_{3}=\sqrt{K_{1}}+\sqrt{K_{2}}, \quad D_{4}=\sqrt{G_{0}}+\sqrt{K_{1}},
\end{aligned}
$$




$$
\begin{aligned}
& D_{5}=-m_{4} D_{4}\left\{b_{0}^{2} m_{4} D_{3} D_{1}+2 b_{1}\left(3 \sqrt{K_{1}}+\sqrt{K_{2}}\right) K_{2} D_{2}\right\}+K+16 m_{2} D_{3}^{2} \sqrt{K_{2}} D_{4} D_{1}^{2}+ \\
& -4 m_{1} \sqrt{K_{2}} D_{2}\left\{2 K_{1}^{5 / 2} M+2 K_{1}^{2}\left\{3 M \sqrt{K_{2}}+\sqrt{G_{0}}(M-2 \mathrm{Ri})\right\}\right\}+ \\
& -K \sqrt{G_{0}}\left(-2 K_{2} R i+2 K_{2} K+K^{2}\right)+\sqrt{K_{1}}\left(2 K_{2}^{3 / 2} \sqrt{G_{0}}(M-2 \mathrm{Ri})+\right. \\
& \left.-\sqrt{K_{2}} \sqrt{G_{0}}(M-4 R i) K-2 K_{2} K^{2}-K^{3}\right)+ \\
& +K_{1}^{3 / 2}\left\{6 K_{2} M+6 \sqrt{K_{2}} \sqrt{G_{0}}(M-2 R i)-K(M-2 K)\right\}+ \\
& +K_{1}\left\{2 K_{2}^{3 / 2} M+6 K_{2} \sqrt{G_{0}}(M-2 \mathrm{Ri})-\sqrt{K_{2}} M K-\sqrt{G_{0}} K\{M-2(\mathrm{Ri}+K)\}\right\}, \\
& D_{6}=4 \sqrt{m_{1}} \sqrt{K_{2}} D_{3} D_{4} D_{2}^{3} r, \\
& D_{7}=\sqrt{K_{1}}\left\{4 K_{1}^{2}+12 K_{1}^{3 / 2} \sqrt{K_{2}}+2 \sqrt{K_{1}} \sqrt{K_{2}}\left(2 K_{2}-3 K\right)+4 K_{1}\left(3 K_{2}-K\right)+K\left(2 K_{2}+K\right)\right\}, \\
& D_{8}=K_{2}\left\{-8 m_{2} D_{3}^{2} D_{4} D_{2}+b_{1} m_{4} \sqrt{K_{1}} D_{4}\left\{2 K_{1}^{3 / 2}+6 K_{1} \sqrt{K_{2}}+\sqrt{K_{1}}\left(6 K_{2}-K\right)+\sqrt{K_{2}}\left(2 K_{2}+K\right)\right\},\right. \\
& D_{9}=2 \sqrt{K_{2}} \sqrt{G_{0}} \mathrm{Ri} K+2 K_{1}^{2}(M+2 K), \\
& D_{13}=b_{0}^{2} m_{4}^{2} D_{3} D_{4} D_{7}-2 D_{2} D_{8}+2 m_{1} D_{3}\left(D_{9}+D_{10}+D_{11}+D_{12}\right), \\
& D_{10}=K_{1}\left\{4 \sqrt{K_{2}} \sqrt{G_{0}}(M-2 \mathrm{Ri})+2 K_{2}(M-2 K)-K(M+2 K)\right\}, \\
& D_{1}^{3 / 2}\left\{2 M \sqrt{K_{2}}+\sqrt{G_{0}}(M-2 \mathrm{Ri}+2 K)\right\}, \\
& D_{2}^{3 / 2} D_{4} D_{2}^{3} .
\end{aligned}
$$

\section{Results and discussion}

In this study, the system of dimensionless governing equations of the unsteady laminar mixed convection boundary layer flow past a vertical wedge has been solved by two distinct methods, namely, the finite difference method for the entire frequency range and the series solution for the low frequency range and the asymptotic series expansion method for the high frequency range. Numerical solutions are carried out for various values of the Richardson number, Ri, Prandtl number, Pr, vortex viscosity parameter, $K$, pressure gradient parameter, $m$, conduction-radiation parameter, $R_{d}$, surface temperature parameter, $\Delta$, magnetic field parameter, $M$, and heat generation or absorption parameter, $\Omega$. 


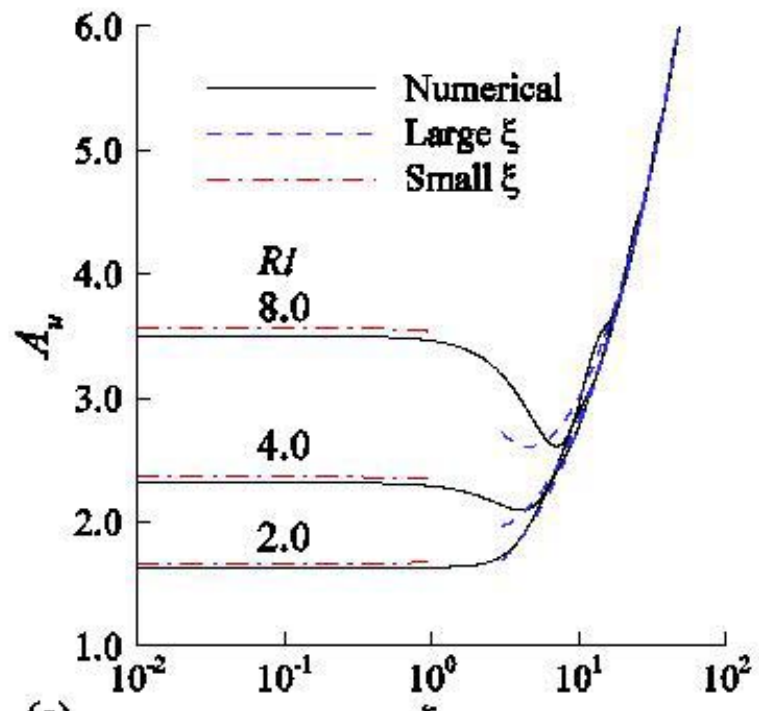

(a)

$\xi$

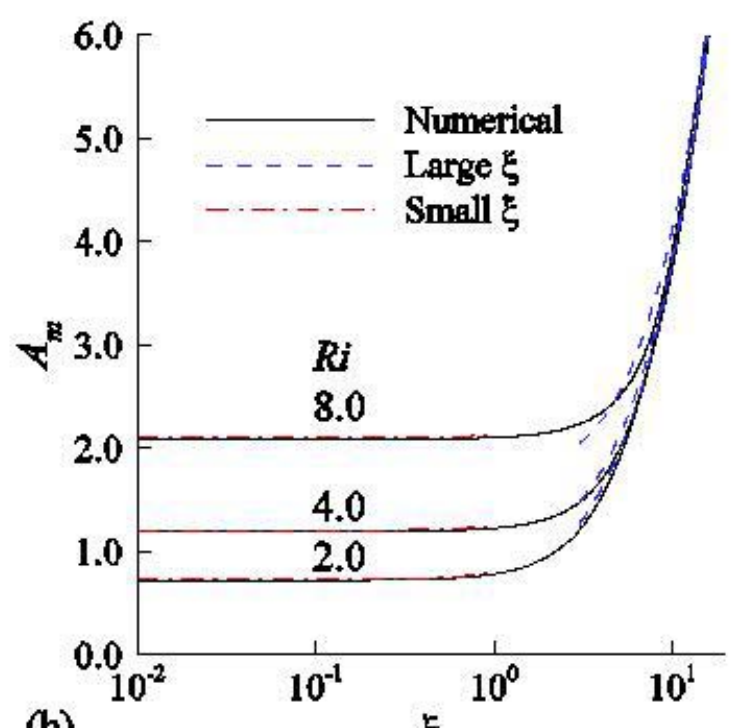

(b)

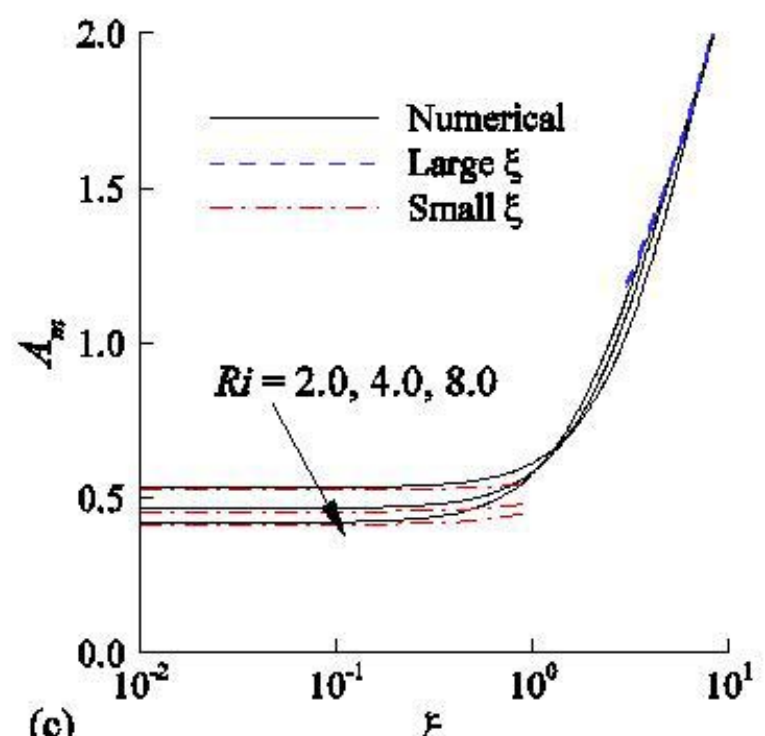

Fig.2. Amplitudes of (a) skin friction (b) couple stress and (c) heat transfer coefficients for different values of Ri while $\operatorname{Pr}=0.73, K=2.0, m=0.3333, R_{d}=0.5, \Delta=0.2, M=0.2$ and $\Omega=0.2$.

In order to validate the numerical solutions, a comparison between the solutions obtained by the finite difference method and the series solutions for small and large $\xi$ is shown in Figs $2 \mathrm{a}-\mathrm{c}$. It is found from the figures that the numerical solutions are in good agreement with the series solutions. Furthermore, the numerical solutions have been compared with Watanabe et al. [5] and Kumari et al. [16]. It should be mentioned that Eqs (2.16) and (2.18) reduce to the corresponding equations of Watanabe et al. [5] and Kumari et al. [16] for Newtonian fluids when $K=0, \Delta=0, M=0, R_{d}=0$ and $\Omega=0$. In this regard, we have made a comparison of the skin friction and heat transfer coefficients in Tab.1 which suggests that the numerical solutions are in excellent agreement. Therefore, our numerical solutions are very accurate. 


\subsection{Effects of different physical parameters on the amplitudes of skin friction, couple stress and heat transfer rates}

Figures $2 \mathrm{a}-\mathrm{c}$ show that the amplitudes of skin friction, couple stress and heat transfer rates increase with the Richardson number, Ri. As the Richardson number, Ri, increases, the buoyancy force becomes prominent and causes the velocity of the fluid to increase. This in turn increases the amplitudes of the skin friction, couple stress and heat transfer rates. In addition, the amplitudes of the skin friction and heat transfer coefficients oscillate near the leading edge and gradually decay in the downstream region.

Table 1. Comparison of the skin friction coefficient, $f^{\prime \prime}(0)$ and heat transfer coefficient, $\theta^{\prime}(0)$, for different $m$ while $\operatorname{Pr}=0.73, \mathrm{Ri}=0.0, K=0.0, R_{d}=0.0, \Delta=0.0, M=0.0$ and $\Omega=0.0$.

\begin{tabular}{lllllll}
\hline$m$ & $f^{\prime \prime}(0)$ & \multicolumn{3}{c}{$\theta^{\prime}(0)$} & & \\
\hline & $\begin{array}{l}\text { Watanabe } \\
\text { et al. }[5]\end{array}$ & $\begin{array}{l}\text { Kumari } \\
\text { et al. }[16]\end{array}$ & $\begin{array}{l}\text { Present } \\
\text { results }\end{array}$ & $\begin{array}{l}\text { Watanabe } \\
\text { et al. }[5]\end{array}$ & $\begin{array}{l}\text { Kumari } \\
\text { et al. }[16]\end{array}$ & $\begin{array}{l}\text { Present } \\
\text { results }\end{array}$ \\
\hline 0 & 0.46960 & 0.46975 & 0.46960 & 0.42015 & 0.42079 & 0.42015 \\
0.0141 & 0.50461 & 0.50472 & 0.50461 & 0.42578 & 0.42635 & 0.42578 \\
0.04350 & 0.56898 & 0.56904 & 0.56897 & 0.43548 & 0.43597 & 0.43548 \\
0.0909 & 0.65498 & 0.65501 & 0.65497 & 0.44730 & 0.44770 & 0.44730 \\
0.1429 & 0.73200 & 0.73202 & 0.73199 & 0.45693 & 0.45728 & 0.45693 \\
0.2000 & 0.80213 & 0.80214 & 0.80211 & 0.46503 & 0.46534 & 0.46503 \\
0.3333 & 0.92765 & 0.92766 & 0.92763 & 0.47814 & 0.47840 & 0.47814 \\
0.5000 & 1.03890 & - & 1.03887 & 0.48848 & - & 0.48848 \\
0.8000 & - & - & 1.17143 & - & - & 0.49951 \\
0.9412 & - & - & 1.21618 & - & - & 0.50295 \\
1.0000 & - & - & 1.23254 & - & - & 0.50418 \\
\hline
\end{tabular}

The effects of the Prandtl number, Pr, on the amplitudes of the skin friction, couple stress and heat transfer rates are shown in Figs $3 \mathrm{a}-\mathrm{c}$. When the Prandtl number increases, the amplitudes of the skin friction and couple stress coefficients decrease while the amplitudes of heat transfer rates increase. Prandtl number is the ratio of momentum to thermal diffusivity of the fluid. Therefore, higher Prandtl number results in reduced thermal boundary layer thickness. A thinner thermal boundary layer corresponds to a large temperature gradient at the wall and hence results in a higher heat transfer coefficient. 

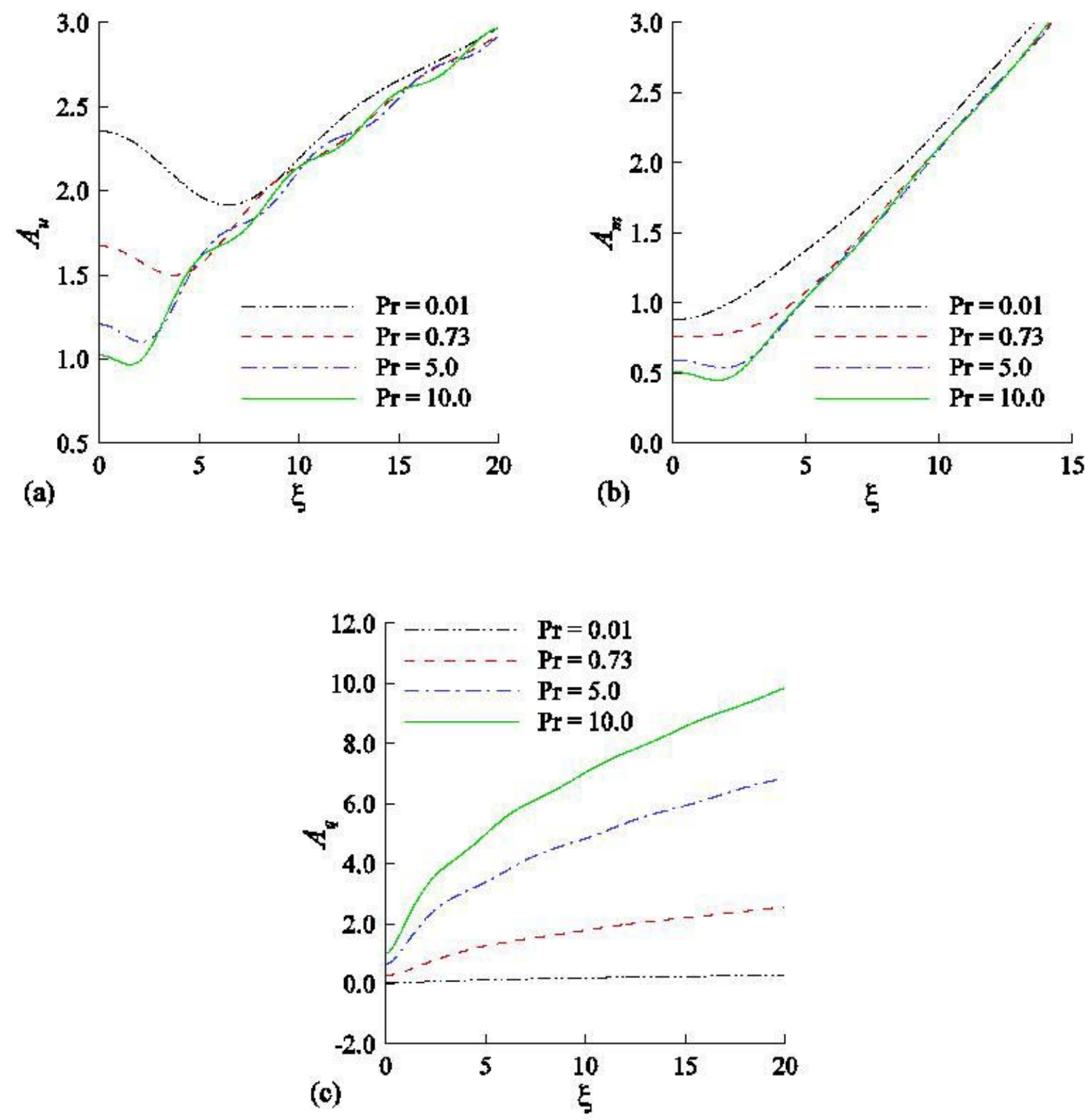

Fig.3. Amplitudes of (a) skin friction (b) couple stress and (c) heat transfer coefficients for different values of Pr while $\mathrm{Ri}=4.0, K=5.0, m=0.3333, R_{d}=0.5, \Delta=0.5, M=0.5$ and $\Omega=0.5$. 

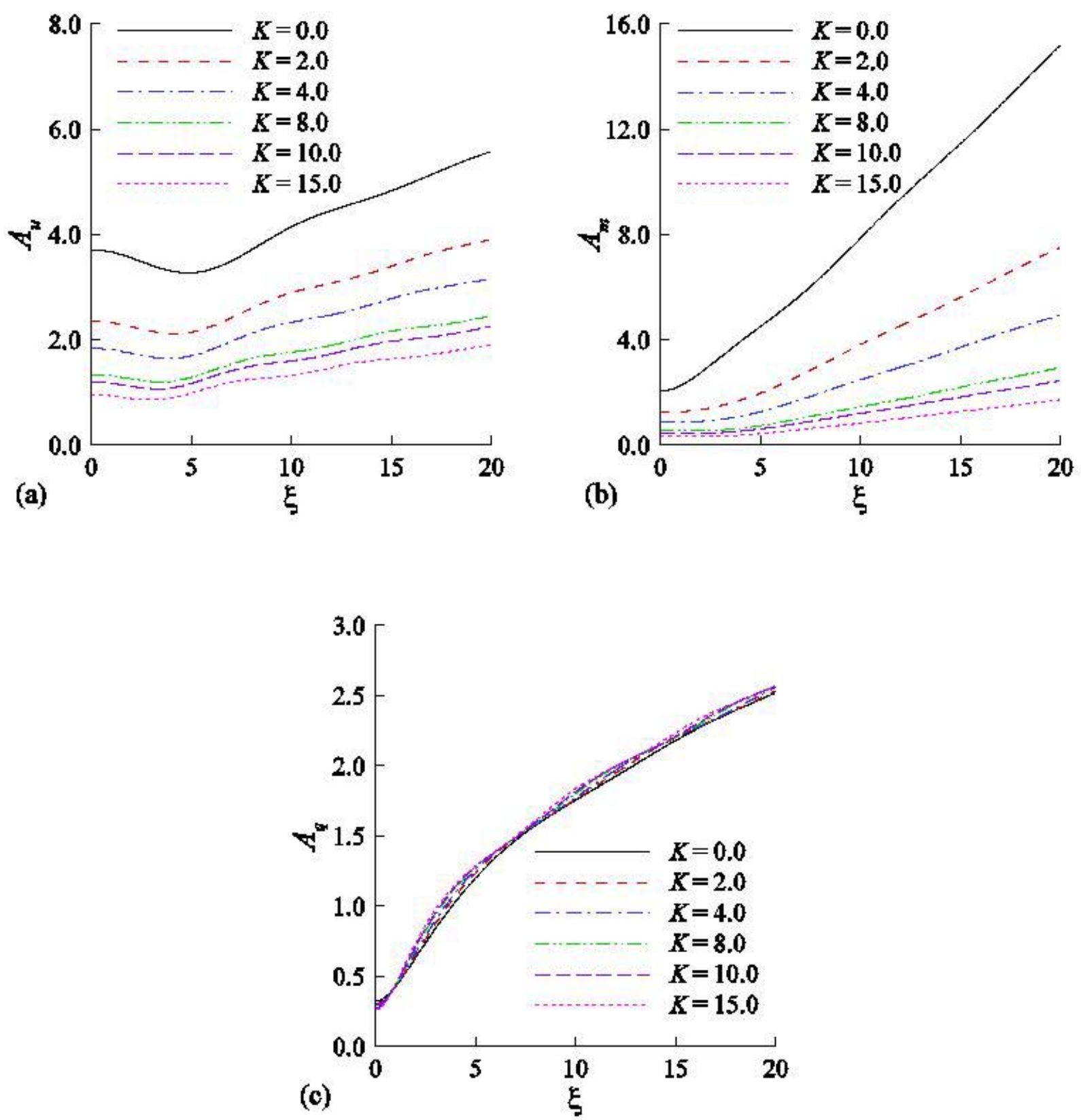

Fig.4. Amplitudes of (a) skin friction (b) couple stress and (c) heat transfer coefficients for different values of $K$ while $\mathrm{Ri}=4.0, \operatorname{Pr}=0.73, m=0.3333, R_{d}=0.5, \Delta=0.5, M=0.5$ and $\Omega=0.5$.

Figures $4 \mathrm{a}-\mathrm{c}$ depict the effects of varying the vortex viscosity parameter, $K$, on the amplitudes of the skin friction, couple stress and heat transfer rates. It is observed that the amplitudes of the skin friction and couple stress coefficients considerably decrease owing to the increase of the vortex viscosity parameter, $K$, while there is a small increase in the amplitudes of the heat transfer coefficient for a large increase of the vortex viscosity parameter, $K$. This is due to the fact that the vortex viscosity parameter $K(=\kappa / \mu)$ is large either for a higher value of the coefficient of vortex viscosity $\kappa$ or for smaller viscosity of the fluid. So the thicknesses of the momentum and microrotation boundary layers increase and the thermal boundary layer decreases for a higher vortex viscosity parameter. 

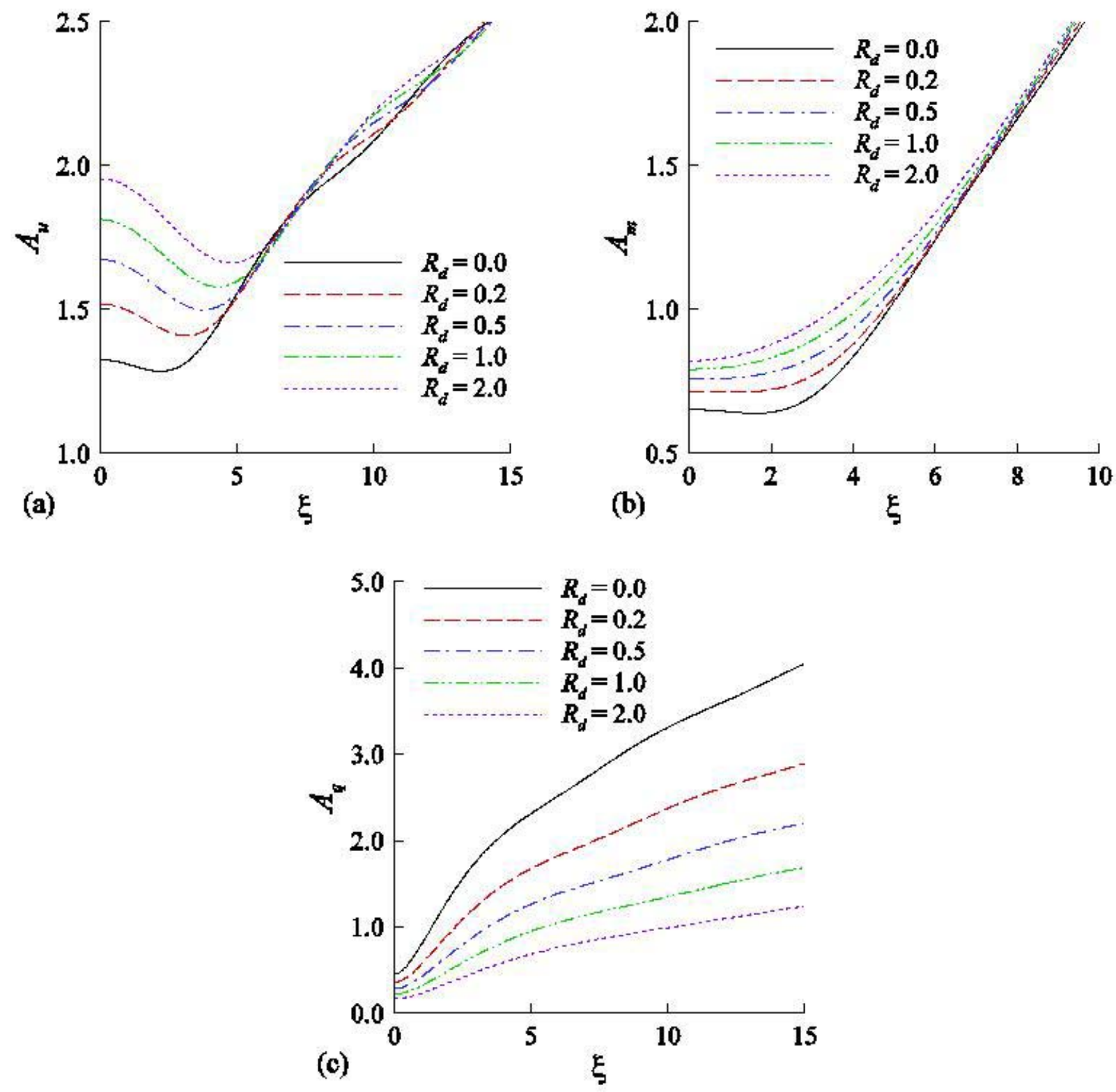

Fig.5. Amplitudes of (a) skin friction (b) couple stress and (c) heat transfer coefficients for different values of $R_{d}$ while $\mathrm{Ri}=4.0, K=5.0, \operatorname{Pr}=0.73, m=0.3333, \Delta=0.5, M=0.5$ and $\Omega=0.5$.

The effects of the conduction-radiation parameter, $R_{d}$, on the amplitudes of the skin friction, couple stress and heat transfer rates are shown in Figs $5 \mathrm{a}-\mathrm{c}$. As the conduction-radiation parameter increases, the amplitudes of skin friction and couple stress increase but the amplitudes of the heat transfer rate decrease. These features are attributed to the decrease of the thicknesses of the momentum and microrotation boundary layers with an increase in $R_{d}$ or the smaller thermal conductivity of the fluid. However, the thermal boundary layer thickness increases as the value of $R_{d}$ increases. When $R_{d}$ is large, radiation dominates heat conduction that lowers the surface temperature of the wedge by dissipating heat into the fluid. For this reason, the temperature difference between the surface and the surrounding fluid is small. It gives a small temperature gradient at the surface and hence a small heat transfer rate. We also observe that the amplitudes of heat 
transfer rate are strongly dependent on the conduction-radiation parameter, $R_{d}$, whereas the effects of $R_{d}$ on the amplitudes of skin friction and couple stress are rather weak.
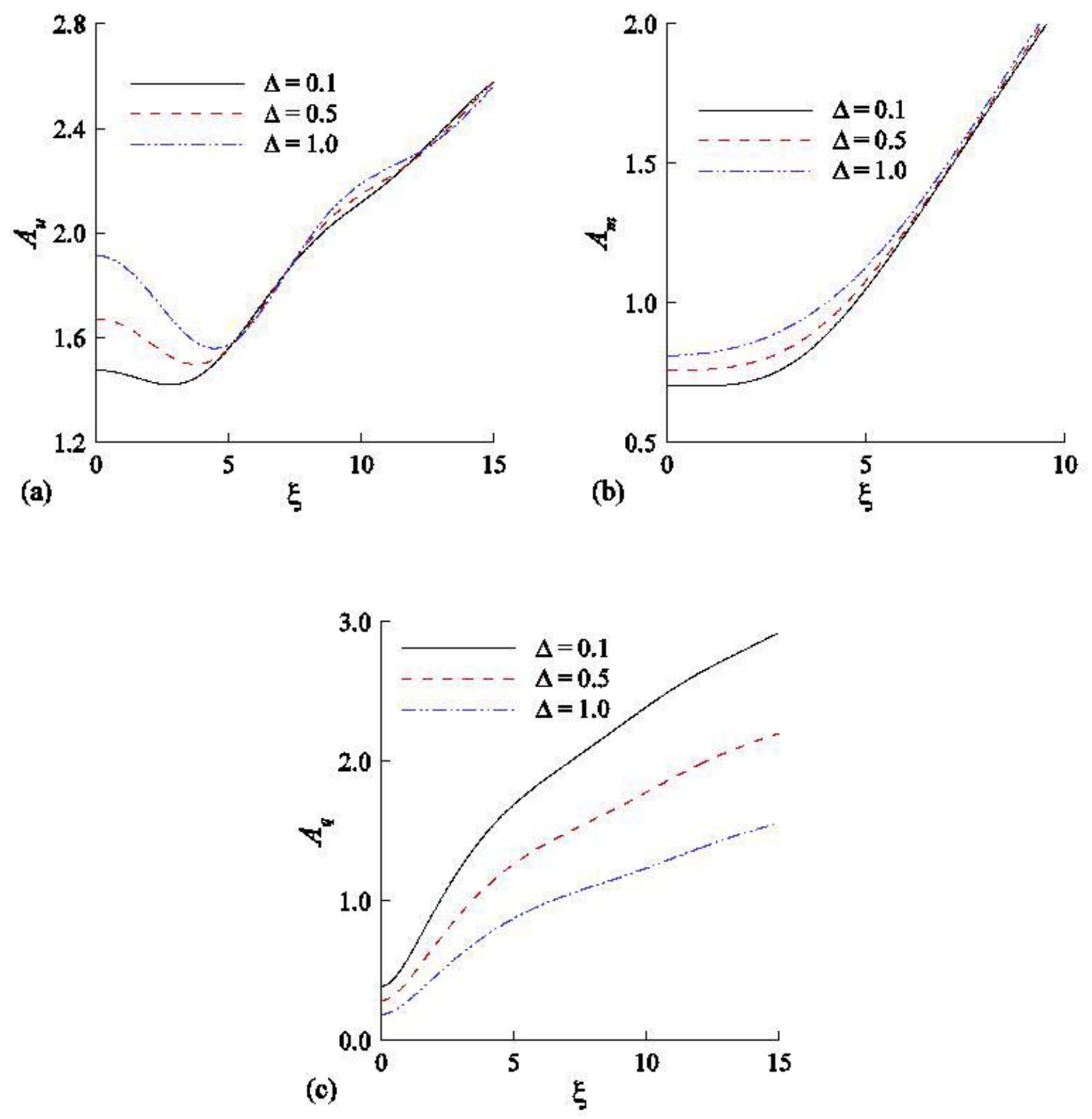

Fig.6. Amplitudes of (a) skin friction (b) couple stress and (c) heat transfer coefficients for different values of $\Delta$ while $\mathrm{Ri}=4.0, K=5.0, \operatorname{Pr}=0.73, m=0.3333, R_{d}=0.5, M=0.5$ and $\Omega=0.5$.

Figures $6 \mathrm{a}-\mathrm{c}$ show the effects of the surface temperature parameter, $\Delta$, on the amplitudes of the skin friction, couple stress and heat transfer rates. It is evident from the figures that the amplitudes of the skin friction and couple stress coefficients increase due to an increase in the surface temperature parameter. When the surface temperature parameter is higher the amplitudes of the heat transfer rate become smaller. The cause of these characteristics is that the higher surface temperature parameter, $\Delta$, reduces the thicknesses of both the momentum and microrotation boundary layers and thickens the thermal boundary layer. 


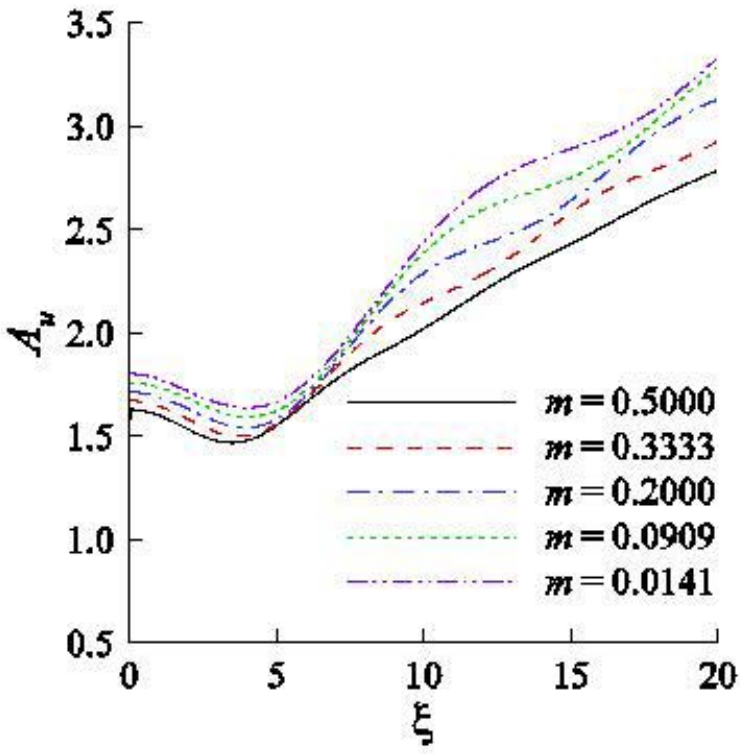

(a)

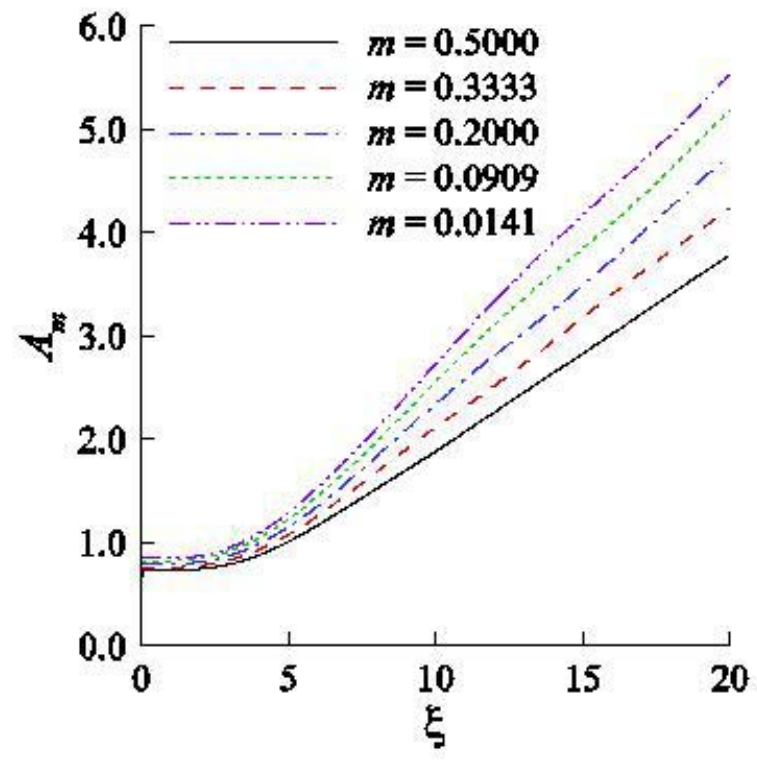

(b)

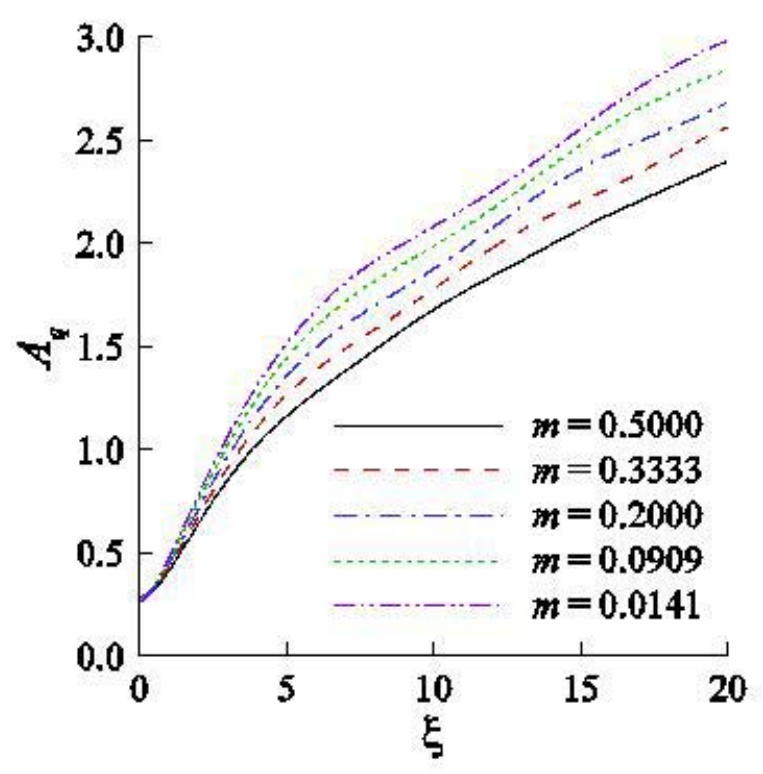

(c)

Fig.7 Amplitudes of (a) skin friction (b) couple stress and (c) heat transfer coefficients for different values of $m$ while $\mathrm{Ri}=4.0, K=5.0, \operatorname{Pr}=0.73, \Delta=0.5, R_{d}=0.5, M=0.5$ and $\Omega=0.5$.

The effects of the pressure gradient parameter, $m$, on the amplitudes of the skin friction, couple stress and heat transfer rates are illustrated in Figs $7 \mathrm{a}-\mathrm{c}$. As the pressure gradient parameter, $m$, increases, the amplitudes of skin friction, couple stress and heat transfer rates decrease. Table 1 shows these results. 

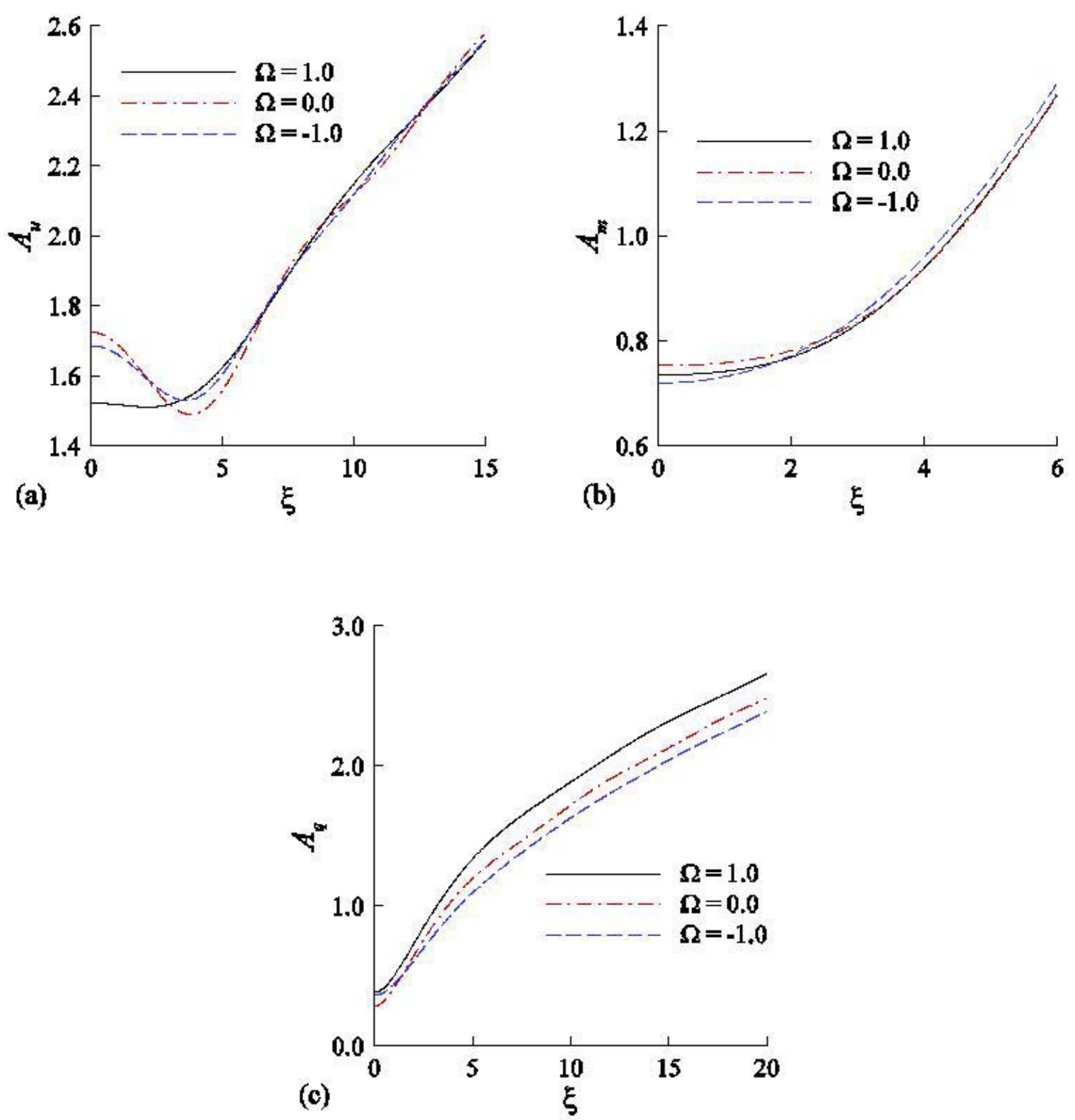

Fig.8. Amplitudes of (a) skin friction (b) couple stress and (c) heat transfer coefficients for different values of $\Omega$ while $\mathrm{Ri}=4.0, K=5.0, \operatorname{Pr}=0.73, m=0.3333, \Delta=0.5, R_{d}=0.5$ and $M=0.5$.

Figures $8 \mathrm{a}$ - c exhibit the effects of heat generation or absorption parameter, $\Omega$, on the amplitudes of skin friction, couple stress and heat transfer rates. It is evident from Fig.8a that in the region $\xi<2$, the amplitudes of the skin friction coefficient relating to heat generation $(\Omega>0)$ are found to be lower than those for heat absorption $(\Omega<0)$. Then the skin friction coefficient increases along $\xi$ with fluctuations. Figure $8 \mathrm{~b}$ shows that the amplitudes of the couple stress coefficient corresponding to heat generation $(\Omega>0)$ are higher than those for heat absorption $(\Omega<0)$ in the region $\xi<2$. Moreover the amplitudes of couple stress for $\Omega<0$ are higher than those for $\Omega>0$. It is found from Fig.8c that the amplitudes of the heat transfer rate corresponding to heat generation $(\Omega>0)$ are larger along the surface of the wedge compared to the values with heat absorption $(\Omega<0)$ and in the absence of this effect $(\Omega=0)$. 

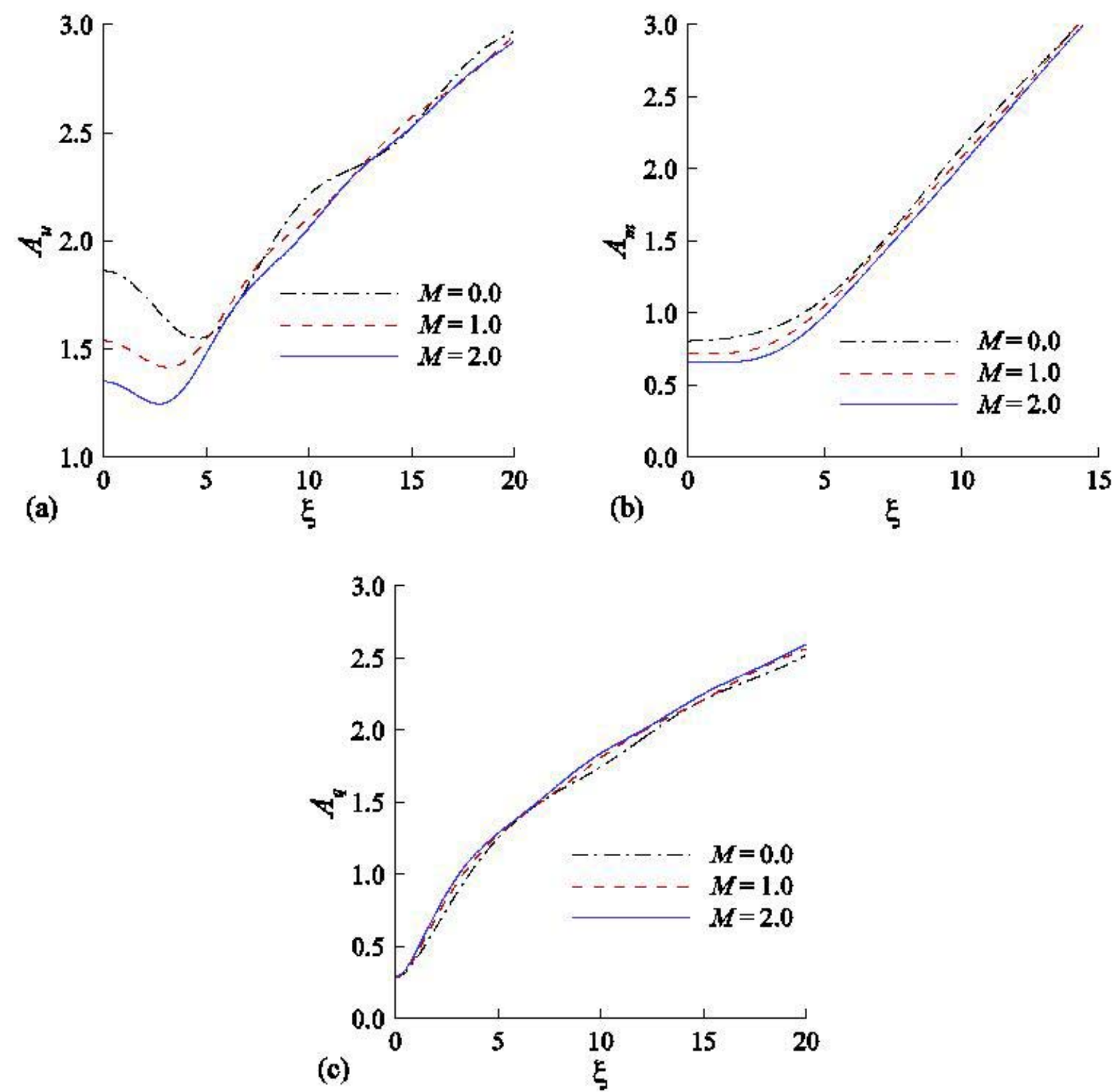

Fig.9. Amplitudes of (a) skin friction (b) couple stress and (c) heat transfer coefficients for different values of $M$ while $\mathrm{Ri}=4.0, K=5.0, \operatorname{Pr}=0.73, m=0.3333, \Delta=0.5, R_{d}=0.5$ and $\Omega=0.5$.

The effects of changing the magnetic field parameter, $M$, on the amplitudes of the skin friction, couple stress and heat transfer coefficients are shown in Figs 9a - c. It is observed from the figures that the amplitudes of the skin friction and couple stress coefficients decrease and the amplitudes of the heat transfer rate increase owing to an increase of the magnetic field parameter, $M$. In addition, for any value of $M$, the amplitudes of the skin friction coefficient first decrease and then increase along $\xi$. The skin friction and heat transfer rates are found to be oscillating in $\xi$ and the amplitudes of oscillations reduce with an increase of $M$. The reasons for the above results can be understood from the definition of the magnetic field parameter, $M\left(=\sigma_{c} B_{0}^{2} x^{1-m} / \rho U_{0}\right)$. The value of $M$ becomes higher either for a higher electrical conductivity, $\sigma_{c}$ or for a higher magnetic field strength, $B_{0}$. The imposition of transverse magnetic field tends to retard the fluid flow. As a result, the thicknesses of momentum and microrotation boundary layers increase and hence the amplitudes of the skin friction and couple stress coefficients decrease with an increase of $M$. The thermal 
boundary layer thickness decreases as the electrical conductivity, $\sigma_{c}$, increases. Consequently, the amplitudes of heat transfer rates increase due to an increase of the magnetic field parameter, $M$.

\subsection{Effects of different physical parameters on the transient skin friction, couple stress and heat transfer coefficients}

Now we illustrate the characteristics of the transient skin friction, $\tau$, transient couple stress, $s$, and transient heat transfer, $q$, with the change of the Richardson number, the vortex viscosity parameter, the pressure gradient parameter and the magnetic field parameter using the following expressions

$$
\begin{aligned}
& \tau=\tau_{s}+A_{u} \cos \left(\omega t+\phi_{u}\right), \\
& s=m_{s}+A_{m} \cos \left(\omega t+\phi_{m}\right), \\
& q=q_{s}+A_{q} \cos \left(\omega t+\phi_{q}\right)
\end{aligned}
$$

where $\tau_{s}=f^{\prime \prime}(0), m_{s}=h^{\prime}(0)$ and $q_{s}=\theta^{\prime}(0)$ are respectively the steady mean skin friction, mean couple stress and mean heat transfer.

Figures $10 \mathrm{a}-\mathrm{c}$ demonstrate the transient skin friction, couple stress and heat transfer rates for different values of $\xi$ against $\omega t$. The results indicate that the amplitudes of oscillation of the transient skin friction, couple stress and heat transfer coefficients increase with $\xi$ in the vicinity of the leading edge. For higher values of Ri this tendency is found to decrease in the case of transient skin friction and couple stress and increase for the transient heat transfer rate.
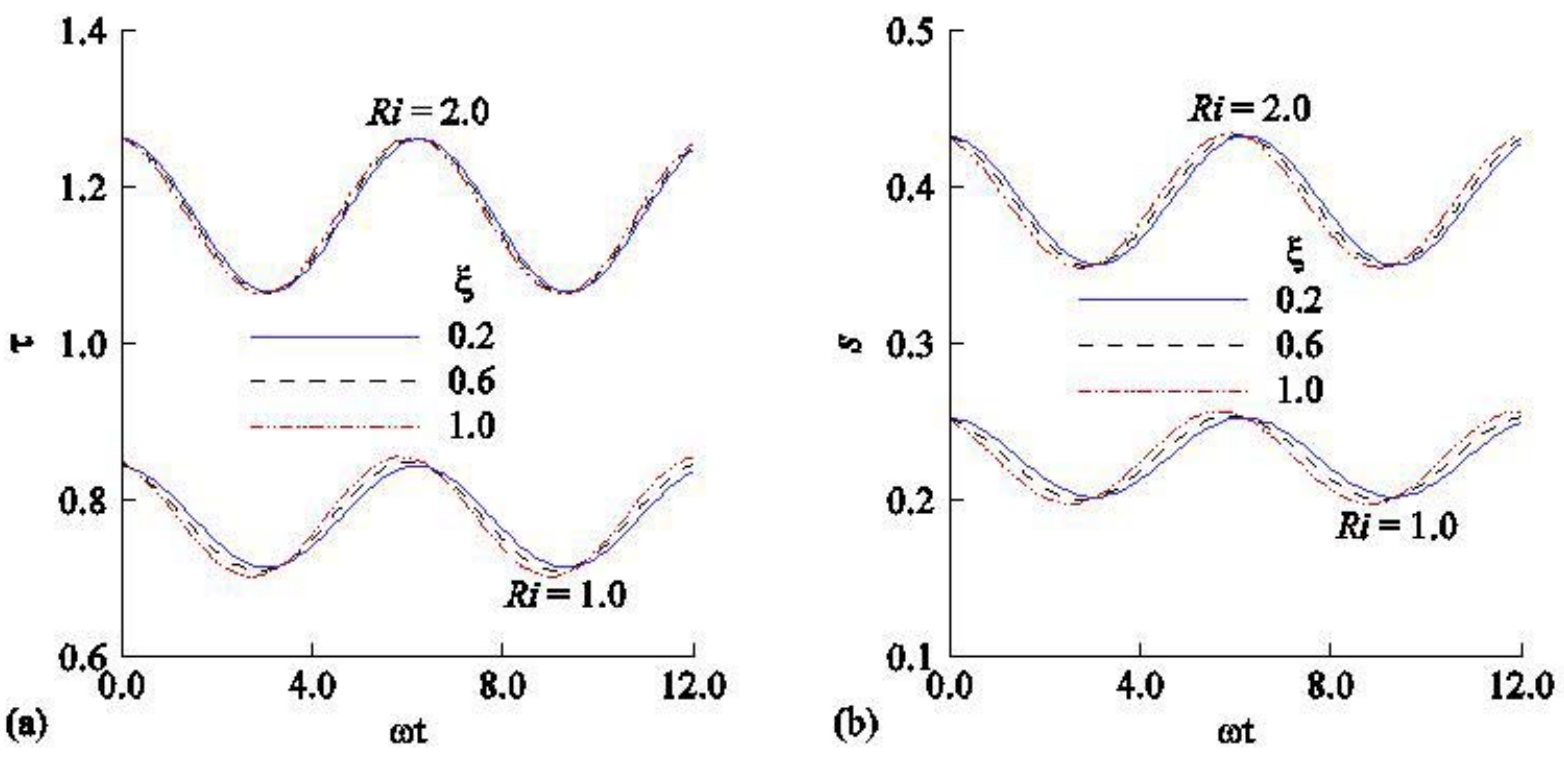


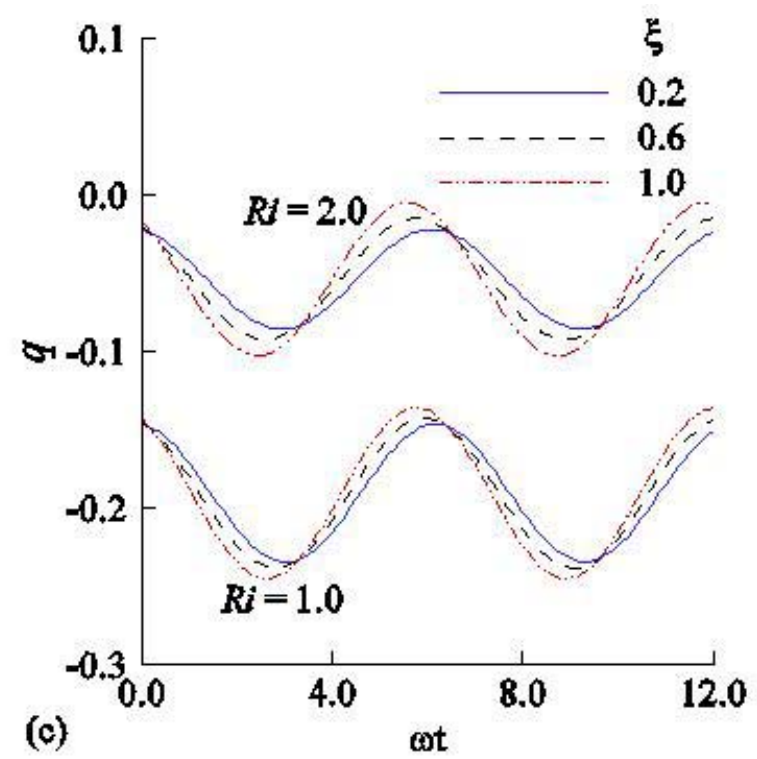

Fig.10. Transient (a) skin friction coefficient (b) couple stress and (3) heat transfer coefficients for different values of $\xi$ against $\omega t$ while $M=1.0, K=5.0, \operatorname{Pr}=0.73, m=0.3333, \Delta=0.5, R_{d}=0.5$ and $\Omega=0.5$.

The variations of the transient skin friction, couple stress and heat transfer rates for different values of $\xi$ against $\omega t$ are shown in Figs 11a - c. It is found from the figures that the amplitudes of oscillation of the transient skin friction, couple stress and heat transfer rate increase owing to an increase of $\xi$. It becomes pronounced for the skin friction and couple stress with higher values of the magnetic field parameter, $M$. On the contrary, Fig.11c shows that higher values of $M$ and $\xi$ reduce the amplitude of oscillation of the heat transfer rate.
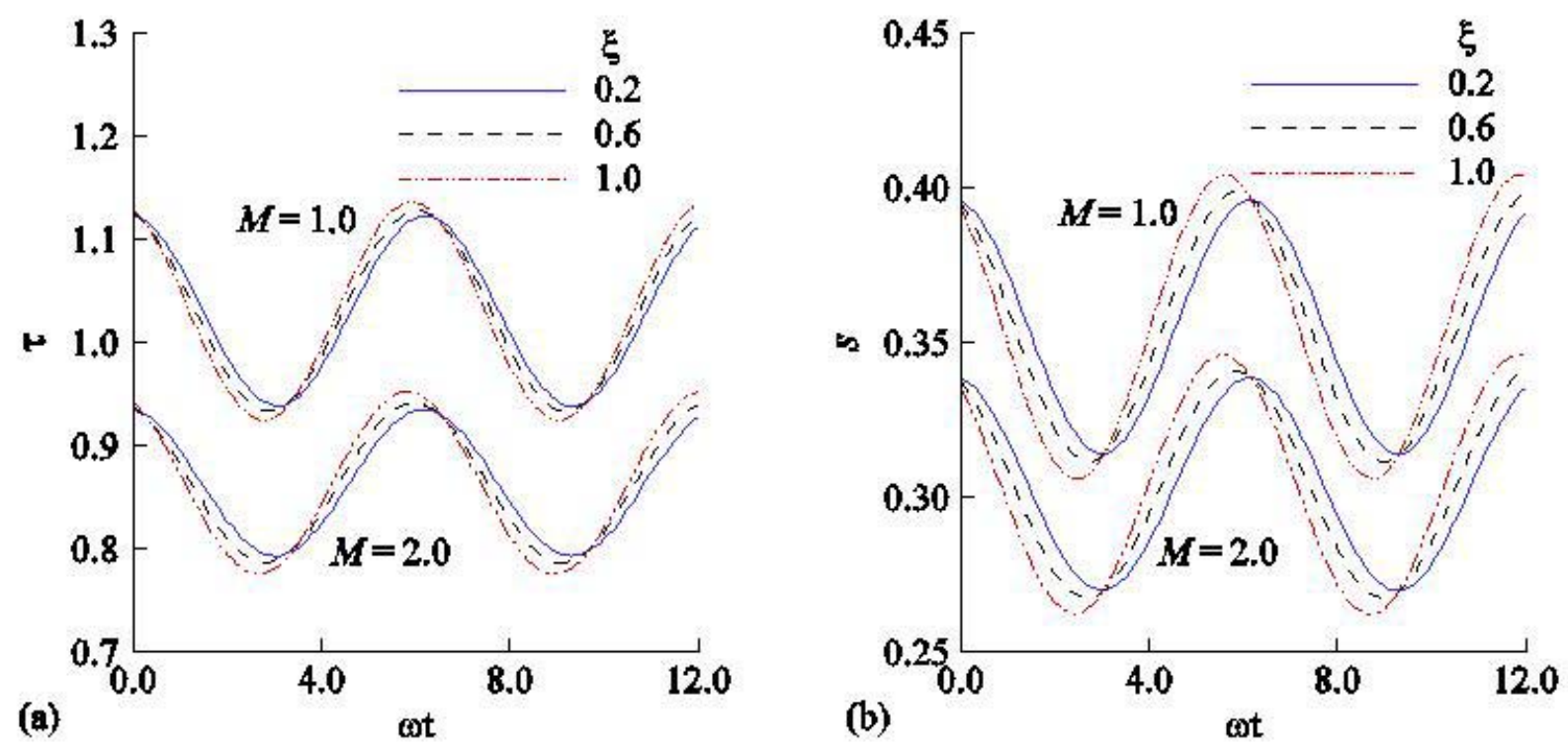


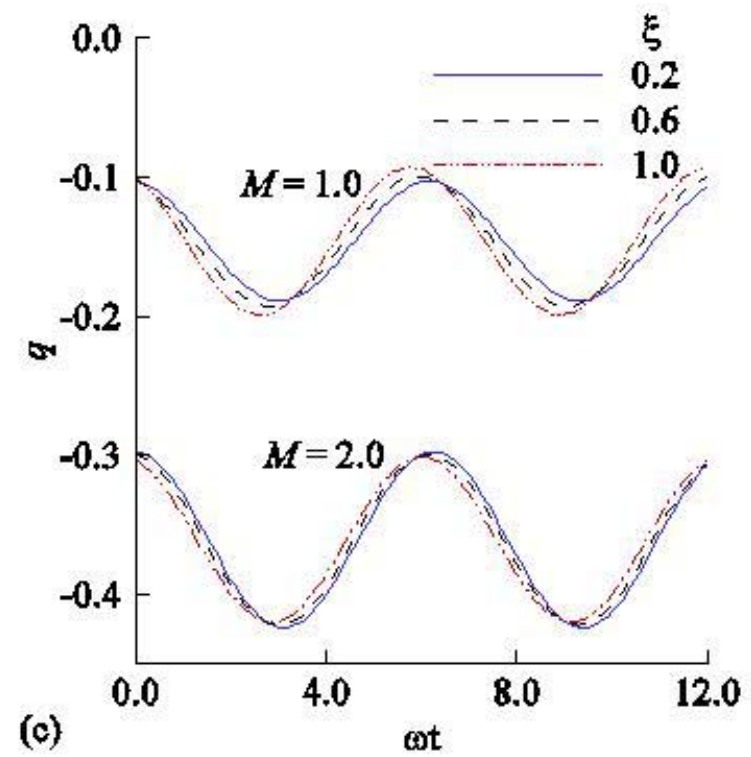

Fig.11. Transient (a) skin friction (b) couple stress and (3) heat transfer coefficients for different values of $\xi$ against $\omega t$ while $\mathrm{Ri}=1.0, K=5.0, \operatorname{Pr}=0.73, m=0.3333, \Delta=0.5, R_{d}=0.5$ and $\Omega=0.5$.

Figures $12 \mathrm{a}-\mathrm{c}$ depict the changes of the transient skin friction, couple stress and heat transfer rates for different values of $\xi$ against $\omega t$. It is seen that the amplitudes of oscillation of the transient skin friction, couple stress and heat transfer rates increase with $\xi$. For increasing values of the mixed convection parameter, $K$, the amplitudes of oscillation with the variation of $\xi$ significantly reduce for the transient skin friction and couple stress coefficients and increase for the transient heat transfer rate.
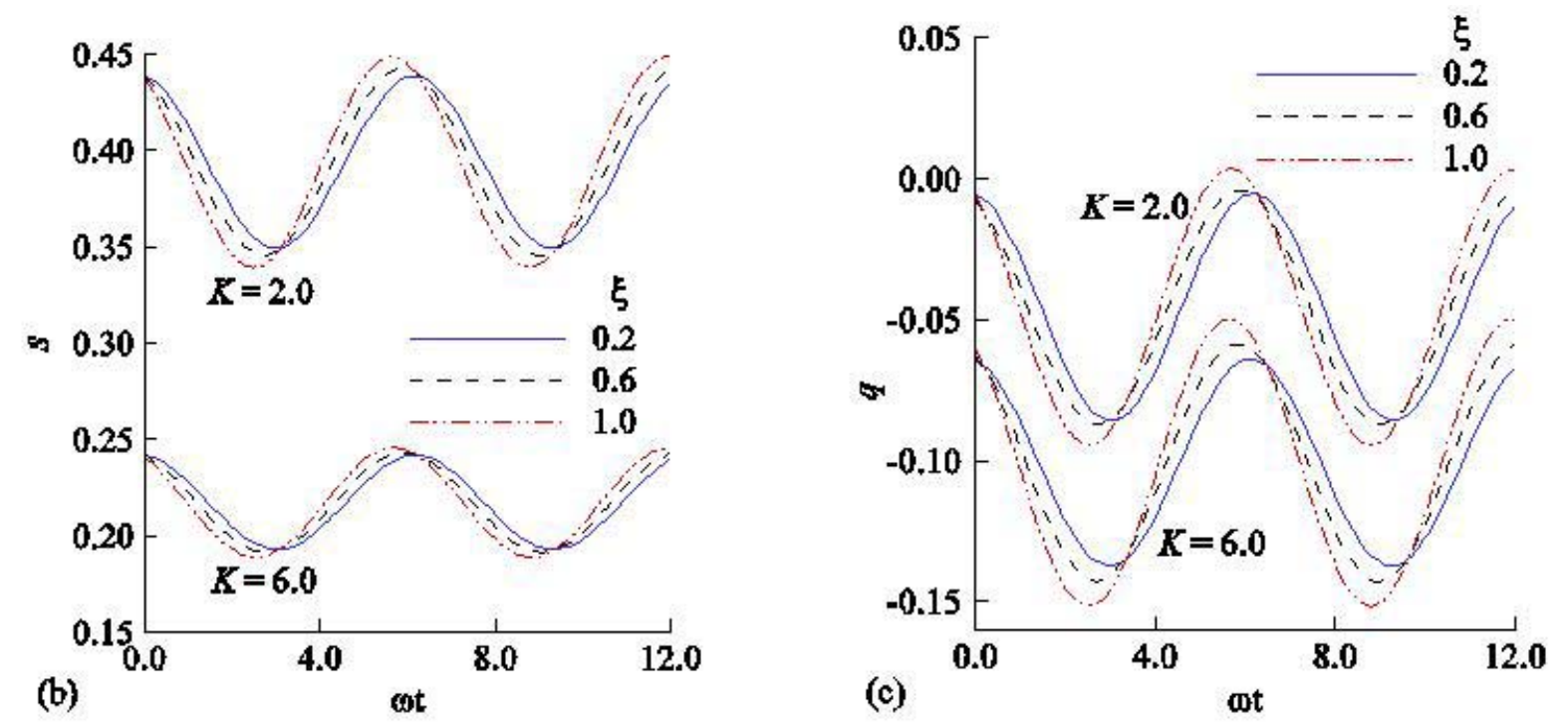

Fig.12. Transient (a) skin friction (b) couple stress and (3) heat transfer coefficients for different values of $\xi$ against $\omega t$ while $\mathrm{Ri}=1.0, M=0.5, \operatorname{Pr}=0.73, m=0.3333, \Delta=0.5, R_{d}=0.5$ and $\Omega=0.5$.

The variations of the transient skin friction, couple stress and heat transfer rate are illustrated for different values of $\xi$ against $\omega t$. Evidently the amplitudes of oscillation of the transient skin friction, couple 
stress and heat transfer rates increase with the distance away from the stagnation point. The amplitudes of oscillation with the change of $\xi$ considerably decrease for higher values of the pressure gradient parameter, $m$, resulting in a decrease of the amplitudes of the skin friction, couple stress and heat transfer rates.
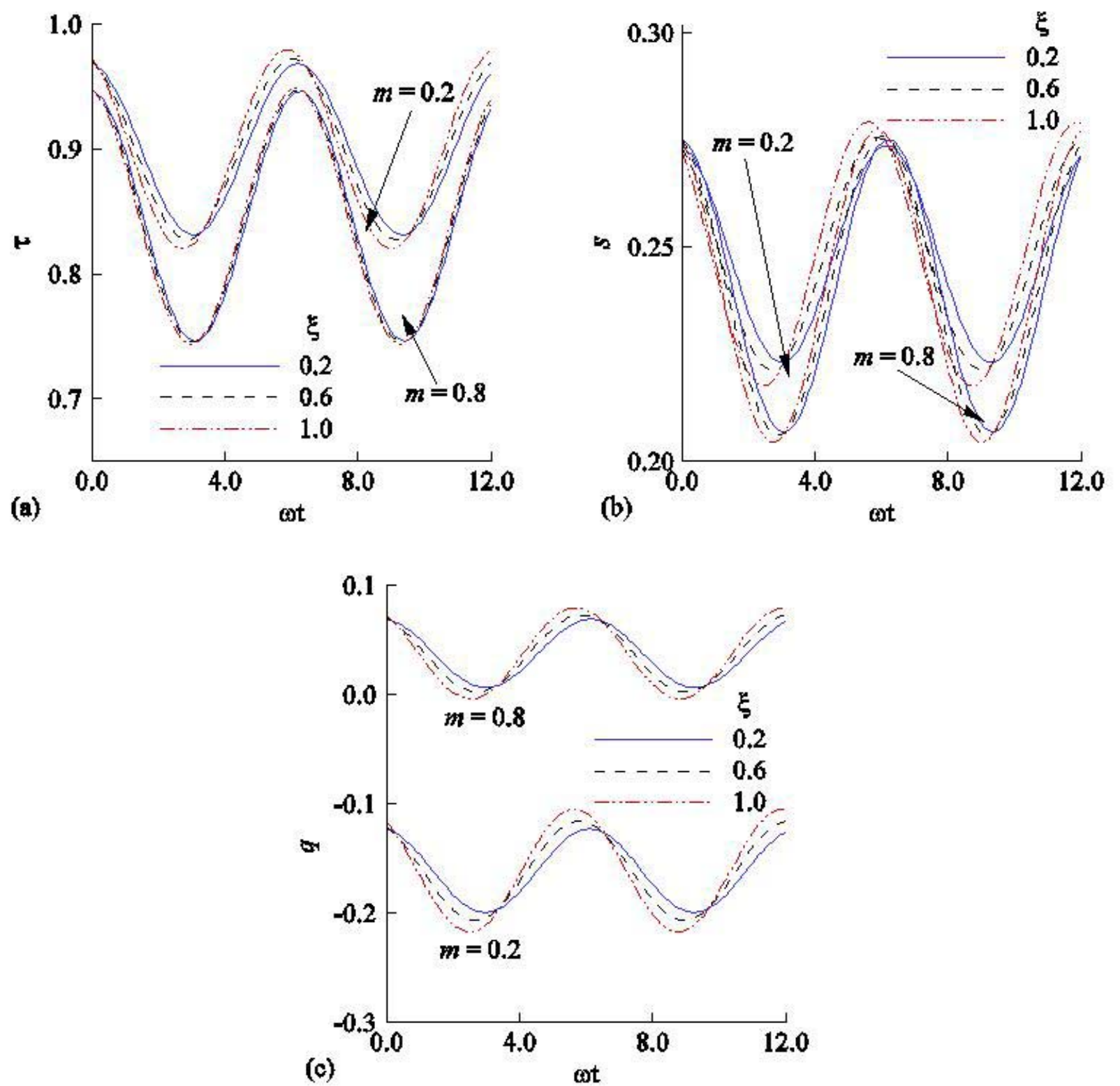

Fig.13. Transient (a) skin friction (b) couple stress and (3) heat transfer coefficients for different values of $\xi$ against $\omega t$ while $\mathrm{Ri}=1.0, M=0.5, \operatorname{Pr}=0.73, K=5.0, \Delta=0.5, R_{d}=0.5$ and $\Omega=0.5$.

\section{Conclusions}

We studied the unsteady MHD mixed convection boundary-layer flow of a micropolar fluid over a vertical wedge in the presence of thermal radiation and heat generation or absorption. The Richardson number and the vortex viscosity parameter significantly affect the amplitudes of the skin friction and couple stress coefficients. However, the amplitudes of the skin friction, couple stress and heat transfer rates are found to be strongly dependent on the Prandtl number, the conduction-radiation parameter, the surface 
temperature parameter and the pressure gradient parameter. In addition, the effects of the magnetic field parameter on the amplitudes of the skin friction coefficient are significant whereas the amplitudes of heat transfer rates are substantially affected by the heat generation or absorption parameter. It is found that the vortex viscosity parameter, which indicates the material property of the micropolar fluid, and the magnetic field parameter significantly reduce the skin friction, however, these bring about a small increase in the rate of heat transfer. As a result, this type of fluid can be used efficiently for controlling the drag force and the rate of heat transfer as it is frequently required in MHD power generators and accelerators and cooling of nuclear reactors. Furthermore, the influences of the Richardson number, the vortex viscosity parameter, the pressure gradient parameter and the magnetic field parameter on the transient skin friction, transient couple stress and transient heat transfer rate are presented. These results reveal how the physical parameters affect the skin friction, couple stress and heat transfer rate near the leading edge of the wedge.

\section{Nomenclature}

$B_{0}$ - strength of magnetic field

$C_{f}$ - local skin friction coefficient

$c_{p}$ - specific heat capacity of the fluid

$F$ - dimensionless stream function of time dependent component

$f$-dimensionless stream function of steady-state component

$\mathrm{Gr}_{x}$ - Grashof number

$g$ - acceleration due to gravity

$j$ - micro-inertia density

$K$ - vortex viscosity parameter

$k$ - coefficient of gyro-viscosity

$k^{*}$ - Rosseland mean absorption coefficient

$M$ - magnetic field parameter

$m$ - pressure gradient parameter

$m_{N}$ - couple stress coefficient

$N$ - component of the micro-rotation vector normal to the $x y$-plane

$\mathrm{Nu}$ - heat transfer rate (Nusselt number)

$\operatorname{Pr}-$ Prandtl number $=v / \alpha$

$q-$ transient heat transfer

$q_{r}$ - radiative heat-flux in the $y$-direction

$R_{d}$ - conduction-radiation parameter

$\operatorname{Re}_{x}-$ Reynolds number $=U_{0} x^{m+1} / v$

$\mathrm{Ri}-$ Richardson's number $=\mathrm{Gr}_{x} / \mathrm{Re}_{x}^{2}$

$s$ - transient couple stress

$T$ - temperature of the fluid

$t$ - time

$U_{0}$-free stream velocity

$u, v$ - fluid velocities in the $x$ - and $y$ - direction respectively

$x, y$-coordinates parallel with and perpendicular to the wedge surface

$\alpha$ - thermal diffusivity

$\beta$ - coefficient of volumetric expansion

$\gamma$ - spin-gradient viscosity

$\Delta$ - surface temperature parameter

$\eta$ - similarity variable

$\theta, \Theta$ - dimensionless temperature function

$\kappa_{c}-$ thermal conductivity

$\mu-$ viscosity of the fluid

$\xi$ - similarity variable

$v$ - kinematic viscosity

$\rho$ - fluid density 
$\sigma_{c}-$ electrical conductivity

$\sigma^{*}$ - Stefan-Boltzman constant

$\tau$ - transient skin friction

$\varphi$ - related to angle of wedge $(\pi \varphi)$

$\psi$ - stream function

$\Omega$ - heat generation $(>0)$ or absorption $(<0)$ parameter

$\omega-$ amplitude of oscillation

\section{Subscripts}

$s-$ refers to the steady state

$w$ - refers to the surface of the wedge

$\infty-$ ambient condition

\section{Superscript}

' - differentiation with respect to $Y$ or $\eta$

\section{References}

[1] Sing P.J., Roy S. and Ravindran R. (2009): Unsteady mixed convection flow over a vertical wedge. - Int. J. Heat Mass Transf., vol.52, pp.415-421.

[2] Lin H.T. and Lin L.K. (1987): Similarity solutions for laminar forced convection heat transfer from wedges to fluids of any Prandtl number. - Int. J. Heat Mass Transf., vol.30, No.6, pp.1111-1118.

[3] Watanabe T. (1990): Thermal boundary layers over a wedge with uniform suction or injection in forced flow. Acta Mech., vol.83, pp.119-126.

[4] Watanabe T. (1991): Forced and free mixed convection boundary layer flow with uniform suction or injection on a vertical flat plate. - Acta Mech., vol.89, pp.123-132.

[5] Watanabe T., Funazaki K. and Taniguchi H. (1994): Theoretical analysis on mixed convection boundary layer flow over a wedge with uniform suction or injection. - Acta Mech., vol.105, pp.133-141.

[6] Lok Y.Y., Amin N. and Pop I. (2003): Unsteady boundary layer flow of a micropolar fluid near the rear stagnation point of a plane surface. - Int. J. Therm. Sci., vol.42, pp.995-1001.

[7] Kim Y.J. (1999): Thermal boundary layer flow of a micropolar past a wedge with constant wall temperature. Acta Mechanica, vol.138, pp.113-121.

[8] Kim Y.J. and Kim T.A. (2003): Convective micropolar boundary layer flows over a wedge with constant surface heat flux. - Int. J. Appl. Mech. Eng., vol.8, pp.147-153, Special issue: ICER 2003.

[9] Mohammadein A.A. and Gorla R.S.R. (1996): Effects of transverse magnetic field on mixed convection in a micropolar fluid on a horizontal plate with vectored mass transfer. - Acta Mech., vol.118, pp.1-12.

[10] Yih K.Y. (1999): MHD forced convection flow adjacent to a non-isothermal wedge. - Int. Comm. Heat Mass Transf., vol.26, No.6, pp.819-827.

[11] Ishak A., Nazar R. and Pop I. (2008): MHD boundary-layer flow of a micropolar fluid past a wedge with variable wall temperature. - Acta Mech., vol.196, pp.75-86.

[12] Ishak A., Nazar R. and Pop I. (2009): MHD boundary layer flow of a micropolar fluid past a wedge with constant wall heat flux. - Commun. Nonlinear Sci. Numer. Simul., vol.14, pp.109-118.

[13] Gorla R.S.R., Takhar H.S. and Slaouti A. (1998): Magnetohydrodynamic free convection boundary layer flow of a thermomicropolar fluid over a vertical plate. - Int. J. Eng. Sci., vol.36, No.3, pp.315-327.

[14] Bhargava R., Kumar L. and TakharH.S. (2003): Numerical solution of free convection MHD micropolar fluid flow between two parallel porous vertical plates. - Int. J. Eng. Sci., vol.41, pp.123-136. 
[15] Nanousis N.D. (1999): Theoretical magnetohydrodynamic analysis of mixed convection boundary-layer flow over a wedge with uniform suction or injection. - Acta Mech., vol.138, pp.21-30.

[16] Kumari M., Takhar H.S. and Nath G. (2001): Mixed convection flow over a vertical wedge embedded in a highly porous medium. - Heat Mass Transfer, vol.37, pp.139-146.

[17] Watanabe T. and Pop I. (1993): Magnetohydrodynamic free convection flow over a wedge in the presence of a transverse magnetic field. - Int. Comm. Heat Mass Transf., vol.20, pp.871-881.

[18] Vajravelu K. and Nayfeh J. (1992): Hydromagnetic convection at a cone and a wedge. - Int. Commun. Heat Mass Transfer, vol.19, pp.701-710.

[19] Ganapathirao M., Ravindran R. and Pop I. (2013): Non-uniform slot suction (injection) on an unsteady mixed convection flow over a wedge with chemical reaction and heat generation or absorption. - Int. J. Heat Mass Transf., vol.67, pp.1054-1061.

[20] Yih K.A. (1999): MHD forced convection flow adjacent to a non-isothermal wedge. - Int. Comm. Heat Mass Transfer, vol.26, pp.819-827.

[21] Chamkha A.J., Mujtaba M., Quadri A. and Issa C. (2003): Thermal radiation effects on MHD forced convection flow adjacent to a non-isothermal wedge in the presence of heat source or sink. - Heat Mass Transf., vol.39, pp.305-312.

[22] Uddin Z., Kumar M. and Harmand S. (2014): Influence of thermal radiation and heat generation/absorption on MHD heat transfer flow of a micropolar fluid past a wedge considering Hall and ion slip currents. - Thermal Sci., vol.18, No.2, pp.489-502.

[23] Raptis A. (1998): Flow of a micropolar fluid past a continuously moving plate by the presence of radiation. Int. J. Heat Mass Transf., vol.41, pp.2865-2866.

[24] Butcher J.C. (1964): Implicit Runge-Kutta Method. - Math. Com., vol.18, pp.50-55.

[25] Naschtsheim P.R. and Sweigert P. (1965): Satisfaction of asymptotic boundary conditions in numerical solution of systems of non-linear equation of boundary layer type. - NASA TN D-3004.

Received: October 10, 2016

Revised: March 20, 2017 